\title{
Atmospheric Oxidation of Propanesulfinic Acid Initiated by OH Radical: Reaction Mechanism, Energetics, Rate Coefficients, and Atmospheric Implications
}

\author{
Parandaman Arathala, and Rabi A. Musah* \\ University at Albany-State University of New York, Department of Chemistry, 1400 Washington \\ Avenue, Albany, NY 12222, USA
}

\section{Supporting Information}

This section contains: Tables S1-S10 present the calculated total electronic energies including zero-point energy corrections, imaginary frequencies of various TSs as discussed in the text at the M06-2X level, rotational constants, vibrational frequencies, optimized geometries of all the reactants, pre-reactive complexes, transition states, post-reactive complexes, and products, $\mathrm{T} 1$ diagnostic values, temperature and pressure dependent rate coefficients, tunneling contributions, and branching ratios. Figures S1-S2 show Potential energy profile for the direct elimination of sulfur dioxide $\left(\mathrm{SO}_{2}\right)$ from PSIA and IRC plot for transition states (TS11). 
Table S1: Calculated total electronic energies for reactants, pre- and post-reactive complexes, transition states (TSs), and products at the M06-2X and CCSD(T) levels. Zero-point energy (ZPE) corrections are given at the M06-2X level.

\begin{tabular}{|c|c|c|c|}
\hline Species & M06-2X/aug-cc-pVTZ & ZPE (M06-2X) & CCSD(T)/aug-cc-pVTZ \\
\hline PSIA & -667.738495 & 0.114376 & -666.911106 \\
\hline$\cdot \mathrm{OH}$ & -75.7338100 & 0.008590 & -75.6455842 \\
\hline $\mathrm{PSIA} \bullet \bullet \mathrm{OH}(\mathrm{RC} 1)$ & -743.480911 & 0.125308 & -742.564127 \\
\hline $\mathrm{PSIA} \bullet \mathrm{OH}(\mathrm{TS} 1)$ & -743.469092 & 0.120361 & -742.553236 \\
\hline PSIA $\bullet O H(P C 1)$ & -743.509668 & 0.123981 & -742.590979 \\
\hline PSIA $\bullet O H(P 1)$ & -667.071050 & 0.100326 & -666.240659 \\
\hline $\mathrm{H}_{2} \mathrm{O}$ & -76.430105 & 0.021547 & -76.3422892 \\
\hline PSIA $\bullet O H(\mathrm{RC} 2)$ & -743.487899 & 0.125928 & -742.569983 \\
\hline $\mathrm{PSIA} \bullet \cdot \mathrm{OH}(\mathrm{TS} 2)$ & -743.473290 & 0.121052 & -742.556560 \\
\hline PSIA $\bullet O H(P C 2)$ & -743.514420 & 0.125132 & -742.594679 \\
\hline PSIA $\bullet \cdot O H(T S 3)$ & -743.475234 & 0.121117 & -742.5585637 \\
\hline PSIA $\bullet O H(P C 3)$ & -743.520338 & 0.124080 & -742.6010463 \\
\hline PSIA $\bullet O H(P 2)$ & -667.076862 & 0.099737 & -666.245529 \\
\hline PSIA $\bullet \cdot O H(T S 4)$ & -743.466375 & 0.120630 & -742.550132 \\
\hline $\mathrm{PSIA} \bullet \bullet \mathrm{OH}(\mathrm{PC} 4)$ & -743.502412 & 0.122839 & -742.584464 \\
\hline PSIA ••OH (P3) & -667.067471 & 0.099248 & -666.237932 \\
\hline PSIA $\bullet O H(\mathrm{RC} 3)$ & -743.476215 & 0.124475 & -742.559203 \\
\hline PSIA $\bullet O H ~(T S 5)$ & -743.468058 & 0.120862 & -742.551821 \\
\hline PSIA $\bullet O H(P C 5)$ & -743.504799 & 0.122866 & -742.586459 \\
\hline PSIA $\bullet \cdot O H(T S 6)$ & -743.471244 & 0.121491 & -742.554233 \\
\hline PSIA ••OH (PC6) & -743.512021 & 0.123932 & -742.593976 \\
\hline PSIA $\bullet O H(R C 4)$ & -743.491789 & 0.126056 & -742.573497 \\
\hline PSIA $\bullet O H(T S 7)$ & -743.482538 & 0.121616 & -742.562762 \\
\hline PSIA $\bullet O H(P C 7)$ & -743.541137 & 0.127485 & -742.625173 \\
\hline PSIA $\bullet O H(P 4)$ & -667.098907 & 0.103123 & -666.272028 \\
\hline PSIA $\bullet O H($ RC5) & -743.489052 & 0.125876 & -742.571922 \\
\hline PSIA $\bullet \cdot O H(T S 8)$ & -743.484679 & 0.125981 & -742.566939 \\
\hline PSIA $\bullet O H(P C 8)$ & -743.495719 & 0.127928 & -742.576969 \\
\hline
\end{tabular}




\begin{tabular}{|c|c|c|c|}
\hline PSIA••OH (TS9) & -743.494514 & 0.126201 & -742.575199 \\
\hline $\mathrm{H}_{2} \mathrm{SO}_{3}$ & -625.058738 & 0.033038 & -624.331056 \\
\hline Propyl radical & -118.442970 & 0.088806 & -118.249573 \\
\hline PSIA••OH (TS10) & -743.485175 & 0.124728 & -742.566593 \\
\hline PSIA (TS11) & -667.633687 & 0.107593 & -666.807045 \\
\hline PSIA (PC9) & -667.738718 & 0.112294 & -666.916322 \\
\hline $\mathrm{SO}_{2}$ & -548.619475 & 0.007277 & -547.990023 \\
\hline Propane & -119.113630 & 0.103888 & -118.922360 \\
\hline
\end{tabular}

Table S2: Imaginary frequencies of all transition states obtained at the M06-2X/aug-cc-pVTZ level of theory.

\begin{tabular}{|c|c|}
\hline Transition State & M06-2X/aug-cc-pVTZ \\
\hline $\mathrm{PSIA} \bullet \bullet \mathrm{OH}(\mathrm{TS} 1)$ & 960 \\
\hline PSIA $\bullet O H ~(T S 2)$ & 1393 \\
\hline PSIA $\bullet O H ~(T S 3)$ & 1087 \\
\hline PSIA $\bullet O H(T S 4)$ & 812 \\
\hline 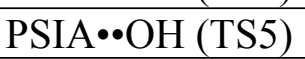 & 744 \\
\hline $\mathrm{PSIA} \bullet \bullet \mathrm{OH}(\mathrm{TS} 6)$ & 1234 \\
\hline 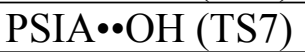 & 2069 \\
\hline $\mathrm{PSIA} \bullet \mathrm{OH}(\mathrm{TS} 8)$ & 105 \\
\hline TS9 & 229 \\
\hline TS10 & 874 \\
\hline $\mathrm{TS} 11\left(\mathrm{SO}_{2}\right)$ & 1915 \\
\hline
\end{tabular}


Table S3: Calculated rotational constants (GHz) for reactants, their complexes, transition states (TS), and products at the M062X/aug-cc-pVTZ level of theory.

\begin{tabular}{|c|c|c|c|}
\hline Species & $\mathbf{A}$ & B & $\mathbf{C}$ \\
\hline PSIA & 6.41 & 1.56 & 1.36 \\
\hline$\cdot \mathrm{OH}$ & 564.35 & & \\
\hline PSIA $\bullet O H(\mathrm{RC} 1)$ & 1.99 & 1.52 & 0.93 \\
\hline PSIA••OH (TS1) & 2.49 & 1.48 & 1.00 \\
\hline $\mathrm{PSIA} \bullet \bullet \mathrm{OH}(\mathrm{PC} 1)$ & 2.61 & 1.32 & 1.04 \\
\hline $\mathrm{PSIA} \bullet \bullet \mathrm{OH}(\mathrm{P} 1)$ & 4.62 & 2.09 & 1.95 \\
\hline $\mathrm{H}_{2} \mathrm{O}$ & 833.94 & 430.91 & 284.11 \\
\hline $\mathrm{PSIA} \bullet \mathrm{OH}(\mathrm{RC} 2)$ & 2.70 & 1.36 & 1.08 \\
\hline PSIA ••OH (TS2) & 2.97 & 1.34 & 1.20 \\
\hline $\mathrm{PSIA} \bullet \bullet \mathrm{OH}(\mathrm{PC} 2)$ & 2.60 & 1.45 & 1.23 \\
\hline $\mathrm{PSIA} \bullet \bullet \mathrm{OH}(\mathrm{TS} 3)$ & 3.01 & 1.34 & 1.03 \\
\hline $\mathrm{PSIA} \bullet \bullet \mathrm{OH}(\mathrm{PC} 3)$ & 2.32 & 1.60 & 1.22 \\
\hline $\mathrm{PSIA} \bullet \mathrm{OH}(\mathrm{P} 2)$ & 4.64 & 2.15 & 1.89 \\
\hline PSIA ••OH (TS4) & 5.23 & 0.74 & 0.68 \\
\hline $\mathrm{PSIA} \bullet \bullet \mathrm{OH}(\mathrm{PC} 4)$ & 3.93 & 0.76 & 0.72 \\
\hline PSIA $\bullet O H(P 3)$ & 6.50 & 1.62 & 1.42 \\
\hline $\mathrm{PSIA} \bullet \mathrm{OH}(\mathrm{RC} 3)$ & 2.88 & 1.08 & 0.84 \\
\hline PSIA••OH (TS5) & 3.63 & 0.96 & 0.81 \\
\hline $\mathrm{PSIA} \bullet \bullet H(\mathrm{PC} 5)$ & 2.56 & 1.40 & 1.10 \\
\hline PSIA••OH (TS6) & 2.96 & 1.39 & 1.15 \\
\hline PSIA ••OH (PC6) & 2.56 & 1.40 & 1.10 \\
\hline PSIA ••OH (RC4) & 2.85 & 1.24 & 1.09 \\
\hline PSIA••OH (TS7) & 3.38 & 1.18 & 1.09 \\
\hline $\mathrm{PSIA} \bullet \bullet \mathrm{OH}(\mathrm{PC} 7)$ & 2.74 & 1.24 & 1.07 \\
\hline $\mathrm{PSIA} \bullet \bullet \mathrm{OH}(\mathrm{P} 4)$ & 7.23 & 1.55 & 1.35 \\
\hline PSIA $\bullet O H($ RC5) & 5.67 & 0.91 & 0.84 \\
\hline PSIA ••OH (TS8) & 4.65 & 1.00 & 0.95 \\
\hline
\end{tabular}




\begin{tabular}{|c|c|c|c|}
\hline PSIA ••OH (PC8) & 4.82 & 1.12 & 1.05 \\
\hline PSIA••OH (TS9) & 4.92 & 1.06 & 0.99 \\
\hline $\mathrm{H}_{2} \mathrm{SO}_{3}$ & 8.62 & 7.84 & 4.96 \\
\hline Propyl radical & 31.19 & 8.99 & 8.05 \\
\hline PSIA $\bullet \bullet H(T S 10)$ & 3.27 & 1.32 & 1.08 \\
\hline PSIA $\bullet O H(T S 11)$ & 5.96 & 1.53 & 1.35 \\
\hline PSIA••OH (PC9) & 4.53 & 1.37 & 1.23 \\
\hline $\mathrm{SO}_{2}$ & 57.76 & 10.42 & 8.83 \\
\hline Propane & 29.46 & 8.54 & 7.54 \\
\hline
\end{tabular}

Table S4: Calculated positive frequencies $\left(\mathrm{cm}^{-1}\right)$ for reactants, their complexes, transition states (TSs) and products at the M062X/aug-cc-pVTZ level of theory.

\begin{tabular}{|l|llllll|}
\hline \multicolumn{1}{c|}{ Species } & \multicolumn{5}{|c|}{ Frequencies } \\
\hline PSIA & 87 & 95 & 158 & 251 & 273 & 306 \\
& 352 & 402 & 460 & 741 & 754 & 780 \\
& 868 & 925 & 1064 & 1066 & 1110 & 1166 \\
& 1193 & 1240 & 1252 & 1330 & 1380 & 1415 \\
& 1451 & 1498 & 1504 & 1512 & 3060 & 3064 \\
& 3077 & 3107 & 3132 & 3141 & 3144 & 3844 \\
\hline $\mathrm{OH}$ & 3771 & & & & & \\
\hline PSIA $\bullet \mathrm{OH}(\mathrm{RC} 1)$ & 32 & 76 & 88 & 110 & 137 & 188 \\
& 246 & 254 & 308 & 359 & 376 & 402 \\
& 444 & 472 & 737 & 752 & 762 & 873 \\
& 926 & 1065 & 1069 & 1110 & 1171 & 1202 \\
& 1246 & 1257 & 1333 & 1378 & 1417 & 1456 \\
& 1498 & 1503 & 1512 & 3060 & 3066 & 3070 \\
\hline
\end{tabular}




\begin{tabular}{|c|c|c|c|c|c|c|}
\hline & 3107 & 3130 & 3140 & 3144 & 3686 & 3841 \\
\hline \multirow{7}{*}{ PSIA••OH (TS1) } & -960 & 55 & 77 & 98 & 112 & 160 \\
\hline & 247 & 265 & 280 & 323 & 344 & 399 \\
\hline & 465 & 639 & 740 & 757 & 774 & 866 \\
\hline & 945 & 971 & 1057 & 1095 & 1114 & 1170 \\
\hline & 1193 & 1237 & 1253 & $3 \quad 1307$ & 1370 & 1400 \\
\hline & 1416 & 1485 & 1504 & 1508 & 3053 & 3068 \\
\hline & 3091 & 3113 & 3138 & 3147 & 3762 & 3837 \\
\hline \multirow[t]{7}{*}{ PSIA••OH (PC1) } & 28 & 65 & 99 & 115 & 140 & 158 \\
\hline & 176 & 239 & 266 & 282 & 299 & 335 \\
\hline & 375 & 403 & 455 & 513 & 703 & 768 \\
\hline & 805 & 957 & 1062 & 1072 & 1135 & 1144 \\
\hline & 1188 & 1242 & 1274 & 1380 & 1410 & 1469 \\
\hline & 1501 & 1512 & 1630 & 3015 & 3060 & 3071 \\
\hline & 3141 & 3158 & 3208 & 3798 & 3833 & 3939 \\
\hline \multirow[t]{6}{*}{ PSIA ••OH $(\mathrm{P} 1)$} & 51 & 75 & 204 & 263 & 280 & 292 \\
\hline & 320 & 418 & 474 & 563 & 677 & 733 \\
\hline & 809 & 929 & 1030 & 1060 & 1131 & 1143 \\
\hline & 1184 & 1262 & 1316 & 1345 & 1403 & 1474 \\
\hline & 1498 & 1505 & 3068 & 3075 & 3119 & 3143 \\
\hline & 3156 & 3217 & 3822 & & & \\
\hline $\mathrm{H}_{2} \mathrm{O}$ & 1619 & 3868 & 3971 & & & \\
\hline \multirow[t]{6}{*}{$\mathrm{PSIA} \bullet \cdot \mathrm{OH}(\mathrm{RC} 2)$} & 51 & 69 & 132 & 138 & 155 & 199 \\
\hline & 262 & 288 & 319 & 355 & 408 & 460 \\
\hline & 474 & 596 & 738 & 765 & 794 & 872 \\
\hline & 927 & 1062 & 1075 & 1111 & 1159 & 1173 \\
\hline & 1241 & 1258 & 1331 & 1380 & 1415 & 1450 \\
\hline & 1498 & 1505 & 1513 & 3061 & 3065 & 3079 \\
\hline
\end{tabular}




\begin{tabular}{|c|c|c|c|c|c|c|}
\hline & 3100 & 3135 & 3145 & 3147 & 3533 & 3840 \\
\hline \multirow[t]{7}{*}{ PSIA $\bullet O H ~(T S 2)$} & -1393 & 75 & 87 & 123 & 156 & 182 \\
\hline & 252 & 263 & 305 & 355 & 396 & 445 \\
\hline & 483 & 645 & 734 & 790 & 806 & 888 \\
\hline & 931 & 953 & 1059 & 1109 & 1111 & 1164 \\
\hline & 1171 & 1203 & 1244 & 1305 & 1377 & 1413 \\
\hline & 1460 & 1487 & 1503 & 15113 & 3047 & 3067 \\
\hline & 3100 & 3129 & 3139 & 31503 & 3679 & 3838 \\
\hline \multirow[t]{7}{*}{$\mathrm{PSIA} \bullet \bullet \mathrm{OH}(\mathrm{PC} 2)$} & 69 & 79 & 89 & 104 & 157 & 198 \\
\hline & 254 & 268 & 301 & 319 & 338 & 351 \\
\hline & 431 & 445 & 512 & 560 & 712 & 746 \\
\hline & 804 & 950 & 1045 & 1060 & 1149 & 1161 \\
\hline & 1169 & 1247 & 1282 & 1379 & 1405 & 1471 \\
\hline & 1499 & 1513 & 1656 & 3010 & 3068 & 3113 \\
\hline & 3140 & 3161 & 3202 & 3779 & 3822 & 3908 \\
\hline \multirow[t]{7}{*}{ 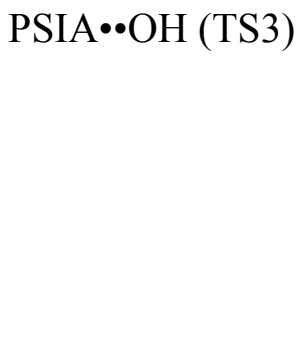 } & -1087 & 44 & 111 & 136 & 157 & 181 \\
\hline & 228 & 274 & 307 & 352 & 410 & 457 \\
\hline & 490 & 659 & 752 & 786 & 808 & 878 \\
\hline & 937 & 1015 & 1085 & 1092 & 1130 & 1170 \\
\hline & 1177 & 1229 & 1254 & 1334 & 1349 & 1398 \\
\hline & 1414 & 1440 & 1491 & 1499 & 3055 & 3065 \\
\hline & 3088 & 3123 & 3129 & 3151 & 3669 & 3839 \\
\hline \multirow[t]{7}{*}{$\mathrm{PSIA} \bullet \bullet \mathrm{OH}(\mathrm{PC} 3)$} & 53 & 66 & 102 & 118 & 128 & 140 \\
\hline & 188 & 207 & 278 & 314 & 337 & 369 \\
\hline & 431 & 455 & 477 & 622 & 646 & 778 \\
\hline & 877 & 917 & 995 & 1071 & 1139 & 1160 \\
\hline & 1171 & 1217 & 1232 & 1389 & 1421 & 1456 \\
\hline & 1479 & 1488 & 1640 & 3012 & 3065 & 3091 \\
\hline & 3140 & 3142 & 3204 & 3679 & 3839 & 3934 \\
\hline
\end{tabular}




\begin{tabular}{|c|c|c|c|c|c|c|}
\hline PSIA ••OH (P2) & $\begin{array}{l}57 \\
382 \\
864 \\
1192 \\
1476 \\
3139\end{array}$ & $\begin{array}{c}99 \\
416 \\
909 \\
1208 \\
1486 \\
3218\end{array}$ & $\begin{array}{l}143 \\
494 \\
998 \\
1235 \\
3023 \\
3842\end{array}$ & $\begin{array}{l}236 \\
526 \\
1066 \\
1387 \\
3072\end{array}$ & $\begin{array}{l}257 \\
597 \\
1137 \\
1413 \\
3088\end{array}$ & $\begin{array}{l}293 \\
770 \\
1171 \\
1458 \\
3128\end{array}$ \\
\hline PSIA ••OH (TS4) & $\begin{array}{l}-812 \\
160 \\
459 \\
922 \\
1218 \\
1418 \\
3090\end{array}$ & $\begin{array}{l}42 \\
265 \\
735 \\
1038 \\
1242 \\
1452 \\
3113\end{array}$ & $\begin{array}{l}52 \\
284 \\
739 \\
1064 \\
1263 \\
1476 \\
3146\end{array}$ & $\begin{array}{l}87 \\
344 \\
777 \\
1072 \\
1326 \\
1504 \\
3159\end{array}$ & $\begin{array}{l}112 \\
357 \\
798 \\
1157 \\
1331 \\
3068 \\
3786\end{array}$ & $\begin{array}{c}129 \\
397 \\
876 \\
1192 \\
1378 \\
3080 \\
3843\end{array}$ \\
\hline PSIA ••OH (PC4) & $\begin{array}{l}9 \\
156 \\
356 \\
780 \\
1191 \\
1469 \\
3152\end{array}$ & $\begin{array}{c}36 \\
180 \\
399 \\
857 \\
1228 \\
1502 \\
3164\end{array}$ & $\begin{array}{l}72 \\
234 \\
457 \\
1013 \\
1231 \\
1610 \\
3267\end{array}$ & $\begin{array}{c}95 \\
267 \\
587 \\
1059 \\
1329 \\
3059 \\
3828\end{array}$ & $\begin{array}{l}103 \\
288 \\
742 \\
1089 \\
1346 \\
3084 \\
3841\end{array}$ & $\begin{array}{l}117 \\
308 \\
752 \\
1155 \\
1448 \\
3115 \\
3947\end{array}$ \\
\hline PSIA ••OH (P3) & $\begin{array}{l}82 \\
347 \\
781 \\
1192 \\
1470 \\
3175\end{array}$ & $\begin{array}{l}95 \\
396 \\
853 \\
1222 \\
1499 \\
3280\end{array}$ & $\begin{array}{l}110 \\
455 \\
1012 \\
1230 \\
3056 \\
3840\end{array}$ & $\begin{array}{l}160 \\
496 \\
1055 \\
1325 \\
3085\end{array}$ & $\begin{array}{l}267 \\
738 \\
1091 \\
1342 \\
3109\end{array}$ & $\begin{array}{l}299 \\
750 \\
1155 \\
1446 \\
3153\end{array}$ \\
\hline PSIA ••OH (RC3) & $\begin{array}{l}38 \\
170 \\
406\end{array}$ & $\begin{array}{l}59 \\
254 \\
461\end{array}$ & $\begin{array}{l}89 \\
271 \\
746\end{array}$ & $\begin{array}{l}103 \\
298 \\
757\end{array}$ & $\begin{array}{l}117 \\
309 \\
780\end{array}$ & $\begin{array}{l}139 \\
353 \\
874\end{array}$ \\
\hline
\end{tabular}




\begin{tabular}{|c|c|c|c|c|c|c|}
\hline & $\begin{array}{l}923 \\
1245 \\
1502 \\
3100\end{array}$ & $\begin{array}{c}1065 \\
1255 \\
1503 \\
3121\end{array}$ & $\begin{array}{c}1067 \\
1332 \\
1518 \\
3134\end{array}$ & $\begin{array}{c}1109 \\
1381 \\
3050 \\
3146\end{array}$ & $\begin{array}{c}1169 \\
1407 \\
3062 \\
3759\end{array}$ & $\begin{array}{c}1190 \\
1449 \\
3082 \\
3844\end{array}$ \\
\hline PSIA••OH (TS5) & $\begin{array}{l}-744 \\
157 \\
459 \\
915 \\
1192 \\
1435 \\
3091\end{array}$ & $\begin{array}{l}40 \\
270 \\
709 \\
1041 \\
1253 \\
1455 \\
3103\end{array}$ & $\begin{array}{l}60 \\
310 \\
749 \\
1068 \\
1304 \\
1483 \\
3150\end{array}$ & $\begin{array}{c}98 \\
326 \\
778 \\
1096 \\
1328 \\
1492 \\
3164\end{array}$ & $\begin{array}{l}118 \\
353 \\
787 \\
1164 \\
1338 \\
3058 \\
3785\end{array}$ & $\begin{array}{l}139 \\
406 \\
884 \\
1181 \\
1388 \\
3080 \\
3844\end{array}$ \\
\hline PSIA••OH (PC5) & $\begin{array}{l}30 \\
164 \\
354 \\
783 \\
1166 \\
1467 \\
3156\end{array}$ & $\begin{array}{l}51 \\
177 \\
410 \\
896 \\
1191 \\
1471 \\
3158\end{array}$ & $\begin{array}{l}86 \\
211 \\
459 \\
986 \\
1252 \\
1609 \\
3261\end{array}$ & $\begin{array}{l}118 \\
275 \\
556 \\
1085 \\
1306 \\
3010 \\
3811\end{array}$ & $\begin{array}{l}133 \\
305 \\
733 \\
1103 \\
1384 \\
3068 \\
3845\end{array}$ & $\begin{array}{l}142 \\
317 \\
770 \\
1158 \\
1450 \\
3083 \\
3941\end{array}$ \\
\hline PSIA••OH (TS6) & $\begin{array}{l}-1234 \\
278 \\
557 \\
940 \\
1168 \\
1425 \\
3092\end{array}$ & $\begin{array}{l}49 \\
328 \\
657 \\
970 \\
1227 \\
1459 \\
3103\end{array}$ & $\begin{array}{l}92 \\
365 \\
739 \\
1079 \\
1262 \\
1468 \\
3153\end{array}$ & $\begin{array}{l}110 \\
378 \\
794 \\
1101 \\
1301 \\
1490 \\
3172\end{array}$ & $\begin{array}{l}168 \\
418 \\
830 \\
1123 \\
1310 \\
3064 \\
3610\end{array}$ & $\begin{array}{l}227 \\
456 \\
895 \\
1166 \\
1386 \\
3081 \\
3837\end{array}$ \\
\hline PSIA ••OH (PC6) & $\begin{array}{l}43 \\
167 \\
416 \\
791\end{array}$ & $\begin{array}{l}57 \\
200 \\
455 \\
855\end{array}$ & $\begin{array}{c}108 \\
270 \\
491 \\
1011\end{array}$ & $\begin{array}{l}131 \\
313 \\
582 \\
1063\end{array}$ & $\begin{array}{l}145 \\
348 \\
729 \\
1093\end{array}$ & $\begin{array}{l}157 \\
389 \\
762 \\
1159\end{array}$ \\
\hline
\end{tabular}




\begin{tabular}{|c|c|c|c|c|c|c|}
\hline & $\begin{array}{l}1161 \\
1458 \\
3156\end{array}$ & $\begin{array}{l}1221 \\
1491 \\
3176\end{array}$ & $\begin{array}{l}1240 \\
1641 \\
3285\end{array}$ & $\begin{array}{l}1329 \\
3063 \\
3680\end{array}$ & $\begin{array}{l}1348 \\
3087 \\
3840\end{array}$ & $\begin{array}{l}1442 \\
3110 \\
3936\end{array}$ \\
\hline PSIA ••OH (RC4) & $\begin{array}{l}52 \\
250 \\
531 \\
924 \\
1245 \\
1498 \\
3106\end{array}$ & $\begin{array}{l}81 \\
307 \\
740 \\
1062 \\
1270 \\
1505 \\
3125\end{array}$ & $\begin{array}{l}112 \\
346 \\
749 \\
1071 \\
1333 \\
1512 \\
3138\end{array}$ & $\begin{array}{c}151 \\
387 \\
768 \\
1109 \\
1381 \\
3049 \\
3143\end{array}$ & $\begin{array}{r}181 \\
415 \\
830 \\
1120 \\
1417 \\
3063 \\
3459\end{array}$ & $\begin{array}{r}221 \\
461 \\
871 \\
1232 \\
1451 \\
3067 \\
3601\end{array}$ \\
\hline 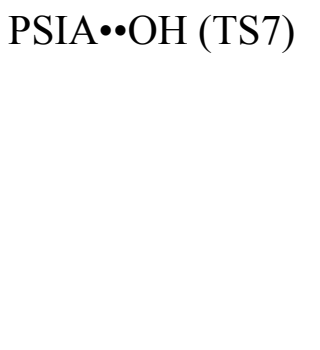 & $\begin{array}{l}-2069 \\
246 \\
601 \\
995 \\
1240 \\
1498 \\
3069\end{array}$ & $\begin{array}{c}63 \\
308 \\
682 \\
1060 \\
1252 \\
1504 \\
3110\end{array}$ & $\begin{array}{c}77 \\
344 \\
742 \\
1072 \\
1329 \\
1512 \\
3131\end{array}$ & $\begin{array}{l}103 \\
354 \\
752 \\
1087 \\
1376 \\
1626 \\
3140\end{array}$ & $\begin{array}{c}130 \\
423 \\
868 \\
1121 \\
1415 \\
3055 \\
3144\end{array}$ & $\begin{array}{c}168 \\
468 \\
924 \\
1126 \\
1450 \\
3064 \\
3754\end{array}$ \\
\hline PSIA ••OH (PC7) & $\begin{array}{l}55 \\
221 \\
418 \\
1057 \\
1282 \\
1505 \\
3118\end{array}$ & $\begin{array}{l}70 \\
249 \\
492 \\
1064 \\
1329 \\
1513 \\
3140\end{array}$ & $\begin{array}{l}100 \\
298 \\
683 \\
1103 \\
1372 \\
1639 \\
3145\end{array}$ & $\begin{array}{c}127 \\
317 \\
746 \\
1126 \\
1417 \\
3066 \\
3157\end{array}$ & $\begin{array}{r}156 \\
340 \\
861 \\
1235 \\
1459 \\
3072 \\
3831\end{array}$ & $\begin{array}{r}163 \\
379 \\
923 \\
1238 \\
1498 \\
3080 \\
3915\end{array}$ \\
\hline PSIA ••OH (P4) & $\begin{array}{l}73 \\
395 \\
1054 \\
1293 \\
1505\end{array}$ & $\begin{array}{l}96 \\
483 \\
1058 \\
1329 \\
1513\end{array}$ & $\begin{array}{c}157 \\
689 \\
1104 \\
1374 \\
3065\end{array}$ & $\begin{array}{c}247 \\
739 \\
1125 \\
1417 \\
3075\end{array}$ & $\begin{array}{c}298 \\
855 \\
1230 \\
1456 \\
3086\end{array}$ & $\begin{array}{c}336 \\
922 \\
1234 \\
1498 \\
3118\end{array}$ \\
\hline
\end{tabular}




\begin{tabular}{|c|c|c|c|c|c|c|}
\hline & 3138 & 3145 & 3158 & & & \\
\hline \multirow[t]{7}{*}{ PSIA ••OH (RC5) } & 36 & 79 & 87 & 127 & 173 & 200 \\
\hline & 252 & 311 & 345 & 368 & 401 & 436 \\
\hline & 478 & 702 & 742 & 762 & 813 & 870 \\
\hline & 924 & 1063 & 1069 & 1116 & 1134 & 1239 \\
\hline & 1246 & 1259 & 1331 & 1381 & 1416 & 1451 \\
\hline & 1498 & 1505 & 1512 & 3062 & 3066 & 3079 \\
\hline & 3107 & 3133 & 3144 & 3145 & 3515 & 3676 \\
\hline \multirow[t]{7}{*}{ PSIA••OH (TS8) } & -105 & 74 & 79 & 94 & 136 & 196 \\
\hline & 250 & 297 & 306 & 350 & 401 & 439 \\
\hline & 534 & 579 & 741 & 751 & 804 & 866 \\
\hline & 924 & 1063 & 1065 & 1116 & 1185 & 1218 \\
\hline & 1239 & 1251 & 1330 & 1378 & 1415 & 1452 \\
\hline & 1498 & 1504 & 1512 & 3062 & 3066 & 3079 \\
\hline & 3110 & 3134 & 3142 & 3147 & 3747 & 3765 \\
\hline \multirow[t]{7}{*}{ PSIA••OH (PC8) } & 71 & 85 & 135 & 172 & 246 & 252 \\
\hline & 277 & 321 & 349 & 405 & 440 & 499 \\
\hline & 519 & 553 & 740 & 830 & 846 & 923 \\
\hline & 999 & 1016 & 1055 & 1115 & 1177 & 1215 \\
\hline & 1226 & 1233 & 1324 & 1362 & 1412 & 1457 \\
\hline & 1497 & 1503 & 1512 & 3061 & 3064 & 3090 \\
\hline & 3110 & 3135 & 3139 & 3166 & 3783 & 3838 \\
\hline \multirow[t]{7}{*}{ PSIA••OH (TS9) } & -229 & 65 & 74 & 115 & 173 & 223 \\
\hline & 251 & 262 & 340 & 398 & 421 & 477 \\
\hline & 520 & 576 & 698 & 772 & 817 & 919 \\
\hline & 925 & 992 & 1064 & 1072 & 1125 & 1183 \\
\hline & 1216 & 1230 & 1320 & 1345 & 1406 & 1466 \\
\hline & 1495 & 1501 & 1512 & 3060 & 3063 & 3106 \\
\hline & 3115 & 3136 & 3137 & 3205 & 3786 & 3834 \\
\hline
\end{tabular}




\begin{tabular}{|l|llllll|}
\hline $\mathrm{H}_{2} \mathrm{SO}_{3}$ & 143 & 345 & 421 & 454 & 501 & 812 \\
& 821 & 1100 & 1124 & 1265 & 3756 & 3760 \\
\hline Propyl radical & 70 & 255 & 334 & 509 & 746 & 894 \\
& 911 & 1042 & 1105 & 1198 & 1312 & 1329 \\
& 1398 & 1471 & 1490 & 1498 & 1510 & 3055 \\
& 3059 & 3091 & 3131 & 3136 & 3168 & 3269 \\
\hline PSIA・・OH (TS10) & -874 & 50 & 79 & 138 & 170 & 248 \\
& 279 & 305 & 343 & 408 & 447 & 469 \\
& 602 & 737 & 742 & 773 & 870 & 922 \\
& 1055 & 1067 & 1100 & 1125 & 1193 & 1206 \\
& 1242 & 1254 & 1332 & 1377 & 1416 & 1448 \\
& 1498 & 1505 & 1512 & 2168 & 3065 & 3072 \\
& 3089 & 3112 & 3138 & 3146 & 3169 & 3879 \\
\hline PSIA・・OH (TS11) & -1915 & 70 & 75 & 153 & 243 & 255 \\
& 326 & 421 & 496 & 605 & 659 & 779 \\
& 894 & 925 & 979 & 1054 & 1075 & 1138 \\
& 1198 & 1225 & 1294 & 1331 & 1360 & 1413 \\
& 1416 & 1490 & 1504 & 1509 & 1783 & 2915 \\
& 3060 & 3070 & 3103 & 3131 & 3138 & 3139 \\
\hline PSIA ••OH (PC9) & 23 & 46 & 69 & 89 & 100 & 147 \\
& 251 & 295 & 372 & 536 & 749 & 891 \\
& 908 & 931 & 1077 & 1181 & 1211 & 1236 \\
& 1317 & 1368 & 1402 & 1413 & 1419 & 1492 \\
& 1497 & 1506 & 1511 & 1520 & 3040 & 3055 \\
& 3065 & 3086 & 3106 & 3121 & 3126 & 3134 \\
\hline $\mathrm{SO}{ }_{2}$ & 536 & 1237 & 1421 & & & \\
& & & & & & \\
\hline & & & & & & \\
& & & & & &
\end{tabular}




\begin{tabular}{|l|lllll|}
\hline Propane $\left(\mathrm{C}_{3} \mathrm{H}_{6}\right)$ & 220 & 279 & 370 & 749 & 891 \\
& 910 & 931 & 1077 & 1182 & 1215 \\
& 1317 & 1366 & 1406 & 1420 & 1493 \\
& 1495 & 1499 & 1511 & 1517 & 3053 \\
& 3054 & 3059 & 3083 & 3115 & 3126 \\
& 3129 & 3130 & & & \\
\hline
\end{tabular}

Table S5: The MO6-2X/aug-cc-pVTZ level optimized geometries of reactants, pre-reactive complexes, transition states, postreactive complexes, and products involved in the PSIA with ${ }^{\circ} \mathrm{OH}$ reaction.

\section{PSIA (stable)}

\begin{tabular}{lrrr}
$\mathrm{S}$ & 0.02077000 & 0.02849700 & 0.08141600 \\
$\mathrm{O}$ & 0.06443500 & 0.07576300 & 1.55307400 \\
$\mathrm{C}$ & 1.74248000 & 0.01821900 & -0.45765000 \\
$\mathrm{H}$ & 1.73721600 & 0.10353500 & -1.54447000 \\
$\mathrm{H}$ & 2.17824400 & 0.91932300 & -0.02342200 \\
$\mathrm{O}$ & -0.20581000 & 1.56748600 & -0.49096900 \\
$\mathrm{H}$ & -1.14711400 & 1.77146900 & -0.44176100 \\
$\mathrm{C}$ & 2.43333900 & -1.24614200 & 0.02647200 \\
$\mathrm{H}$ & 1.92556400 & -2.12210200 & -0.38369900 \\
$\mathrm{H}$ & 2.33853300 & -1.30135300 & 1.11113400 \\
$\mathrm{C}$ & 3.90254400 & -1.26172000 & -0.37861200 \\
$\mathrm{H}$ & 4.39434100 & -2.16688400 & -0.02620600 \\
$\mathrm{H}$ & 4.01149800 & -1.21884000 & -1.46272500 \\
$\mathrm{H}$ & 4.42885600 & -0.40585800 & 0.04452100 \\
& & & \\
OH & & & \\
\hline $\mathrm{O}$ & 0.00000000 & 0.00000000 & -0.00249600 \\
$\mathrm{H}$ & 0.00000000 & 0.00000000 & 0.96938100
\end{tabular}




\section{$\underline{\text { PSIA } \bullet O H ~(R C 1) ~}$}

$\begin{array}{lrrr}\mathrm{S} & -1.07060600 & -0.13233600 & 0.54121600 \\ \mathrm{O} & -0.88357900 & 0.46731600 & 1.87058800 \\ \mathrm{C} & 0.57701900 & -0.26524800 & -0.18022100 \\ \mathrm{H} & 0.45125300 & -0.57686300 & -1.21861800 \\ \mathrm{H} & 0.98993700 & 0.74532700 & -0.14816400 \\ \mathrm{O} & -1.50372100 & 1.08588700 & -0.52295400 \\ \mathrm{H} & -2.44511500 & 1.26421000 & -0.41105900 \\ \mathrm{C} & 1.41502100 & -1.24745600 & 0.62380000 \\ \mathrm{H} & 0.93491600 & -2.22868200 & 0.62073200 \\ \mathrm{H} & 1.45008200 & -0.91077300 & 1.66012200 \\ \mathrm{C} & 2.82412600 & -1.35644300 & 0.05303000 \\ \mathrm{H} & 3.42433800 & -2.05555900 & 0.63275300 \\ \mathrm{H} & 2.80262500 & -1.70327700 & -0.98018100 \\ \mathrm{H} & 3.32299700 & -0.38750100 & 0.06927700 \\ \mathrm{O} & 0.52266900 & 1.57339500 & -2.54493400 \\ \mathrm{H} & -0.29253200 & 1.67602500 & -2.01711600\end{array}$

\section{PSIA $\bullet \circ O H$ (TS1)}

$\begin{array}{lrrr}\mathrm{S} & 0.02341000 & -0.00607100 & 0.12942100 \\ \mathrm{O} & 0.03844000 & 0.26105300 & 1.57709100 \\ \mathrm{C} & 1.75114800 & -0.02298400 & -0.36704200 \\ \mathrm{H} & 1.73446600 & 0.02004100 & -1.54844700 \\ \mathrm{H} & 2.19540500 & 0.90650400 & -0.00838400 \\ \mathrm{O} & -0.24097300 & 1.43375200 & -0.66536600 \\ \mathrm{H} & -1.18104600 & 1.64191800 & -0.60076900 \\ \mathrm{C} & 2.46477800 & -1.27810700 & 0.07536400 \\ \mathrm{H} & 1.93815300 & -2.15251000 & -0.31238600 \\ \mathrm{H} & 2.41850300 & -1.33309100 & 1.16624000 \\ \mathrm{C} & 3.91422400 & -1.28408300 & -0.39732600 \\ \mathrm{H} & 4.42133600 & -2.19241400 & -0.07745500 \\ \mathrm{H} & 3.96416700 & -1.22748800 & -1.48448000\end{array}$




$\begin{array}{lrrr}\mathrm{H} & 4.45703400 & -0.43135600 & 0.01030800 \\ \mathrm{O} & 1.49787600 & 0.24001800 & -2.87225000 \\ \mathrm{H} & 0.88864400 & 0.98577200 & -2.73855800\end{array}$

\section{PSIA $\cdot 0$ OH (PC1)}

$\begin{array}{lrrr}\mathrm{S} & -0.88979800 & 0.49360300 & 1.08823300 \\ \mathrm{O} & -0.93838300 & 1.93251500 & 1.38709700 \\ \mathrm{C} & 0.77409400 & 0.12124700 & 0.71329300 \\ \mathrm{H} & 0.91938500 & 1.16978300 & -2.87849300 \\ \mathrm{H} & 1.45335300 & 0.94493700 & 0.88833100 \\ \mathrm{O} & -1.42017500 & 0.26343100 & -0.49414800 \\ \mathrm{H} & -2.37693000 & 0.13877600 & -0.47840100 \\ \mathrm{C} & 1.20353400 & -1.24303800 & 0.31962900 \\ \mathrm{H} & 0.58414800 & -1.58321500 & -0.51569800 \\ \mathrm{H} & 1.01115000 & -1.94070500 & 1.14370000 \\ \mathrm{C} & 2.67479900 & -1.28349400 & -0.07717400 \\ \mathrm{H} & 2.97125700 & -2.29424800 & -0.35113500 \\ \mathrm{H} & 2.84081700 & -0.63103000 & -0.93218000 \\ \mathrm{H} & 3.30820900 & -0.95475900 & 0.74673200 \\ \mathrm{O} & 0.81861300 & 0.37059900 & -2.35794400 \\ \mathrm{H} & -0.02591900 & 0.46416900 & -1.90018000\end{array}$

\section{$\mathrm{CH}_{3}-\mathrm{CH}_{2}-\mathrm{C} \cdot \mathrm{H}-\mathrm{S}(\mathrm{O}) \mathrm{OH}(\mathrm{P1})$}

$\begin{array}{lrrr}\mathrm{S} & -1.10689400 & 0.03597400 & -0.04167700 \\ \mathrm{O} & -0.46833600 & 0.18884000 & 1.27793800 \\ \mathrm{C} & -0.24940400 & -1.26173400 & -0.85067000 \\ \mathrm{H} & -0.60730000 & -1.51195200 & -1.83925900 \\ \mathrm{O} & -0.50580300 & 1.22019100 & -1.05391000 \\ \mathrm{H} & -1.05569200 & 2.00685400 & -0.94708100 \\ \mathrm{C} & 0.96931900 & -1.83826100 & -0.23704400 \\ \mathrm{H} & 1.13803900 & -2.83678600 & -0.63984200 \\ \mathrm{H} & 0.81858600 & -1.92327700 & 0.83993400\end{array}$




$\begin{array}{llrr}\mathrm{C} & 2.19684600 & -0.94563400 & -0.48965500 \\ \mathrm{H} & 3.08174000 & -1.39017100 & -0.03620600 \\ \mathrm{H} & 2.37611200 & -0.82311300 & -1.55704200 \\ \mathrm{H} & 2.03503100 & 0.03747300 & -0.05160700\end{array}$

\section{$\underline{\mathbf{H}}_{2} \underline{\mathbf{O}}$}

$\begin{array}{lrrr}\mathrm{H} & -0.00144100 & 0.00000000 & -0.00128000 \\ \mathrm{O} & 0.00060700 & 0.00000000 & 0.95809000 \\ \mathrm{H} & 0.92638800 & 0.00000000 & 1.20973300\end{array}$

\section{$\underline{\text { PSIA } \bullet O H ~(R C 2) ~}$}

S $\quad-1.28766000 \quad-0.08059900 \quad-0.01601700$

$\begin{array}{llll}\mathrm{O} & -0.93441600 & 0.52232700 & 1.29287600\end{array}$

$\begin{array}{llll}\mathrm{C} & 0.28080700 & -0.35453600 & -0.85932600\end{array}$

$\mathrm{H} \quad \begin{array}{llll}\mathrm{H} & 0.03886200 & -0.66966500 & -1.87418400\end{array}$

$\mathrm{H} \quad 0.76294500 \quad 0.62431700 \quad-0.88216600$

$\begin{array}{lllll}\mathrm{O} & & -1.70907700 & 1.13837400 & -1.04285100\end{array}$

$\begin{array}{lllll}\mathrm{H} & & -2.63713700 & 1.35718700 & -0.89571700\end{array}$

C $\quad 1.11867200 \quad-1.38136800-0.11197100$

$\mathrm{H} \quad 0.62963700 \quad-2.35688900 \quad-0.15298900$

$\mathrm{H} \quad \begin{array}{llll}\mathrm{H} & 1.18292300 & -1.09909900 & 0.94031600\end{array}$

$\begin{array}{llll}\mathrm{C} & 2.52137900 & -1.46362800 & -0.70199900\end{array}$

$\mathrm{H} \quad 3.11632400 \quad-2.21240800 \quad-0.18215200$

$\mathrm{H} \quad 2.48968700 \quad-1.72981200 \quad-1.75908900$

$\begin{array}{llll}\mathrm{H} & 3.02559100 & -0.50237000 & -0.60462400\end{array}$

$\begin{array}{llll}\mathrm{O} & 1.77653400 & 1.20380200 & 1.42166400\end{array}$

$\begin{array}{llll}\mathrm{H} & 0.80050600 & 1.17118500 & 1.54148900\end{array}$

\section{PSIA $\bullet$ OH (TS2)}

$\begin{array}{lrrr}\mathrm{S} & 0.08579700 & 0.13642700 & 0.21653900 \\ \mathrm{O} & 0.29377600 & 0.49360300 & 1.63635200 \\ \mathrm{C} & 1.73188900 & -0.01833700 & -0.47757400\end{array}$




$\begin{array}{rrr}1.69075700 & 0.08904400 & -1.56083000 \\ 2.28791700 & 0.95491900 & -0.03344000 \\ -0.22962800 & 1.51565400 & -0.62775200 \\ -1.16349700 & 1.73328500 & -0.51984300 \\ 2.45227700 & -1.24763000 & 0.02428800 \\ 1.94282800 & -2.14475800 & -0.33906600 \\ 2.39932400 & -1.26307800 & 1.11403400 \\ 3.90809300 & -1.24765100 & -0.42946100 \\ 4.42614800 & -2.13179900 & -0.06261300 \\ 3.97818000 & -1.24108500 & -1.51751600 \\ 4.41888300 & -0.36435500 & -0.04826000 \\ 2.79571700 & 1.77441200 & 0.88053200 \\ 2.08596300 & 1.65719900 & 1.54114900\end{array}$

\section{PSIA • - OH (PC2)}

$\begin{array}{lrrr}\mathrm{S} & -1.31952800 & -0.09341400 & -0.07691300 \\ \mathrm{O} & -0.97400600 & 0.06028200 & 1.35285100 \\ \mathrm{C} & 0.08365400 & -0.76921600 & -0.87153300 \\ \mathrm{H} & 0.05287500 & -0.72707700 & -1.95259200 \\ \mathrm{H} & 1.02707000 & 2.02453100 & 0.12882700 \\ \mathrm{O} & -1.16531200 & 1.38864200 & -0.82263900 \\ \mathrm{H} & -2.01950600 & 1.83627700 & -0.76861800 \\ \mathrm{C} & 1.08063800 & -1.57895600 & -0.13395700 \\ \mathrm{H} & 0.82963200 & -2.64282400 & -0.22679800 \\ \mathrm{H} & 1.02413100 & -1.33055400 & 0.92581600 \\ \mathrm{C} & 2.49477200 & -1.33982500 & -0.66699300 \\ \mathrm{H} & 3.20640500 & -1.97491900 & -0.14204400 \\ \mathrm{H} & 2.55580300 & -1.57041300 & -1.73084000 \\ \mathrm{H} & 2.77268600 & -0.29960000 & -0.51186100 \\ \mathrm{O} & 1.48817300 & 1.53397700 & 0.81449100 \\ \mathrm{H} & 0.77251700 & 1.12043600 & 1.31592000\end{array}$




\section{$\underline{\text { PSIA } \bullet \bullet H ~(T S 3) ~}$}

S $\quad-0.00473800 \quad-0.00457300 \quad 0.04203700$

$\begin{array}{llll}\mathrm{O} & -0.07370500 & -0.09395800 & 1.51578600\end{array}$

$\begin{array}{llll}\mathrm{C} & 1.75037400 & 0.02672700 & -0.37982000\end{array}$

$\mathrm{H} \quad 1.79779100 \quad 0.20889000 \quad-1.45546700$

$\mathrm{H} \quad 2.16620500 \quad 0.88408500 \quad 0.15222100$

$\begin{array}{lllll}\mathrm{O} & -0.19332100 & 1.57742300 & -0.38801800\end{array}$

$\mathrm{H} \quad-1.13661800 \quad 1.77909300 \quad-0.40755000$

$\begin{array}{llll}\mathrm{C} & 2.42885000 & -1.26547500 & 0.01190500\end{array}$

$\mathrm{H} \quad \begin{array}{llll}\mathrm{H} & 1.87698000 & -2.14429500 & -0.32595500\end{array}$

$\mathrm{H} \quad 2.41873100 \quad-1.33026100 \quad 1.19593400$

C $\quad 3.88986200 \quad-1.30751100-0.38115100$

$\mathrm{H} \quad 4.35785000 \quad-2.22548300 \quad-0.03166300$

$\mathrm{H} \quad 4.00305500 \quad-1.25728600 \quad-1.46644500$

$\mathrm{H} \quad 4.42502800 \quad-0.46506600 \quad 0.05647400$

$\begin{array}{llll}\mathrm{O} & 2.42429300 & -1.06593300 & 2.52572400\end{array}$

$\mathrm{H} \quad 1.52830200 \quad-0.67693500 \quad 2.53799500$

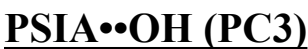

$\begin{array}{lrrr}\mathrm{S} & -1.00587100 & -0.07787700 & -0.46714600 \\ \mathrm{O} & -0.71064000 & -0.88056600 & 0.74402300 \\ \mathrm{C} & 0.63171800 & 0.40533900 & -1.15227300 \\ \mathrm{H} & 0.40082500 & 1.10812000 & -1.95176100 \\ \mathrm{H} & 1.10935500 & 0.91314100 & -0.31088200 \\ \mathrm{O} & -1.34558000 & 1.46497300 & 0.01955000 \\ \mathrm{H} & -2.28120100 & 1.51628200 & 0.24879900 \\ \mathrm{C} & 1.36792500 & -0.78798500 & -1.60065200 \\ \mathrm{H} & 1.20355300 & -1.13937600 & -2.60959700 \\ \mathrm{H} & 1.36694700 & 0.67628900 & 2.82741500 \\ \mathrm{C} & 2.14185900 & -1.62130300 & -0.64657700 \\ \mathrm{H} & 2.88784500 & -2.22384900 & -1.16259600 \\ \mathrm{H} & 2.63123000 & -1.00248300 & 0.10658200\end{array}$




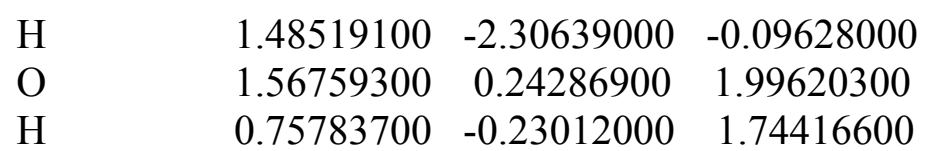

\section{$\mathrm{CH}_{3}-\mathrm{C} \cdot \mathrm{H}-\mathrm{CH}_{2}-\mathrm{S}(\mathbf{O}) \mathrm{OH}(\mathbf{P 2})$}

$\begin{array}{lrrr}\mathrm{S} & -0.89207100 & 0.39376500 & 0.19701900 \\ \mathrm{O} & -0.61241800 & -0.74668200 & 1.08515600 \\ \mathrm{C} & 0.64671800 & 0.64230300 & -0.82464700 \\ \mathrm{H} & 0.61574100 & -0.21663000 & -1.49708000 \\ \mathrm{H} & 0.45433500 & 1.56068900 & -1.37779300 \\ \mathrm{O} & -0.62079200 & 1.79157500 & 1.04514500 \\ \mathrm{H} & -1.39376300 & 1.95803100 & 1.59743700 \\ \mathrm{C} & 1.85580900 & 0.67983400 & -0.00120300 \\ \mathrm{H} & 2.13206500 & 1.62617700 & 0.43958600 \\ \mathrm{C} & 2.49978300 & -0.56360500 & 0.48990600 \\ \mathrm{H} & 2.02732600 & -0.90370200 & 1.41826600 \\ \mathrm{H} & 2.39185100 & -1.37627400 & -0.22987500 \\ \mathrm{H} & 3.55987800 & -0.40949100 & 0.69091700\end{array}$

\section{PSIA $\bullet$ OH (TS4)}

$\begin{array}{lrrr}\mathrm{S} & 0.12605700 & 0.09803200 & 0.22167100 \\ \mathrm{O} & 0.40535700 & 0.40418000 & 1.63457700 \\ \mathrm{C} & 1.74470300 & -0.05853300 & -0.56671400 \\ \mathrm{H} & 1.57180300 & -0.18591600 & -1.63510300 \\ \mathrm{H} & 2.23633300 & 0.89852500 & -0.38682600 \\ \mathrm{O} & -0.18043800 & 1.51552500 & -0.57829900 \\ \mathrm{H} & -1.10581900 & 1.74863400 & -0.43795900 \\ \mathrm{C} & 2.50665700 & -1.22438300 & 0.04860100 \\ \mathrm{H} & 1.96395300 & -2.15633000 & -0.12036500 \\ \mathrm{H} & 2.56091500 & -1.07348800 & 1.12720300 \\ \mathrm{C} & 3.89694200 & -1.32859300 & -0.54025300 \\ \mathrm{H} & 4.43244000 & -2.26210200 & -0.07910100\end{array}$




$\begin{array}{rrr}3.90106500 & -1.52793000 & -1.61136300 \\ 4.52510500 & -0.46849900 & -0.31436000 \\ 4.93238300 & -3.31260400 & 0.69857200 \\ 5.01686800 & -2.82512700 & 1.53336500\end{array}$

\section{PSIA $\bullet O H$ (PC4)}

$\begin{array}{lrrr}\mathrm{S} & -1.44060100 & 0.56112900 & 0.23985100 \\ \mathrm{O} & -1.05254800 & 1.36680900 & 1.41011700 \\ \mathrm{C} & 0.04744800 & 0.44287300 & -0.78014500 \\ \mathrm{H} & -0.23056900 & -0.06758100 & -1.70132900 \\ \mathrm{H} & 0.32420600 & 1.47462600 & -0.99826500 \\ \mathrm{O} & -2.17813700 & 1.55229300 & -0.86438600 \\ \mathrm{H} & -3.10480400 & 1.64681500 & -0.61416100 \\ \mathrm{C} & 1.14104100 & -0.30114200 & -0.00703800 \\ \mathrm{H} & 0.79399000 & -1.31016900 & 0.22898700 \\ \mathrm{H} & 1.30656900 & 0.22239300 & 0.93473700 \\ \mathrm{C} & 2.39626900 & -0.36877000 & -0.80393300 \\ \mathrm{H} & 3.03746200 & -2.39552100 & 0.31971000 \\ \mathrm{H} & 2.47534000 & -1.06608800 & -1.62585900 \\ \mathrm{H} & 3.12740700 & 0.42357500 & -0.74264500 \\ \mathrm{O} & 2.57855600 & -3.23387400 & 0.43476100 \\ \mathrm{H} & 3.13066300 & -3.75567000 & 1.02069200\end{array}$

\section{${\mathrm{C} \cdot \mathrm{H}_{2}}_{2}-\mathrm{CH}_{2}={ }^{-\mathrm{CH}_{2}}=$}

$\begin{array}{lccc}\mathrm{S} & -1.44745300 & 0.55334800 & 0.23236700 \\ \mathrm{O} & -1.06442500 & 1.33316300 & 1.42163400 \\ \mathrm{C} & 0.04398900 & 0.45367500 & -0.78405600 \\ \mathrm{H} & -0.23005800 & -0.04219400 & -1.71436600 \\ \mathrm{H} & 0.32172000 & 1.48834900 & -0.98611100 \\ \mathrm{O} & -2.18158500 & 1.56675000 & -0.85339000 \\ \mathrm{H} & -3.10740600 & 1.66194000 & -0.60013600 \\ \mathrm{C} & 1.14054300 & -0.29777800 & -0.02098800\end{array}$




$\begin{array}{lrrr}\mathrm{H} & 0.78563500 & -1.30389500 & 0.21454400 \\ \mathrm{H} & 1.30647200 & 0.22104900 & 0.92391800 \\ \mathrm{C} & 2.39460400 & -0.36385600 & -0.81693100 \\ \mathrm{H} & 2.54978800 & -1.15169100 & -1.53783100 \\ \mathrm{H} & 3.09378700 & 0.45790100 & -0.80272300\end{array}$

\section{PSIA $\bullet 0 H$ OHC3)}

$\begin{array}{lrrr}\mathrm{S} & -1.38641900 & 0.40111800 & 0.67528400 \\ \mathrm{O} & -0.90052500 & 1.30595900 & 1.73242000 \\ \mathrm{C} & 0.09020500 & -0.24226400 & -0.13609900 \\ \mathrm{H} & -0.24130900 & -0.84001700 & -0.98514200 \\ \mathrm{H} & 0.62277300 & 0.63540500 & -0.50496900 \\ \mathrm{O} & -1.79199700 & 1.30712800 & -0.65092200 \\ \mathrm{H} & -2.68177100 & 1.65395400 & -0.51719700 \\ \mathrm{C} & 0.91550200 & -1.04249200 & 0.85824600 \\ \mathrm{H} & 0.32392900 & -1.87677700 & 1.24222200 \\ \mathrm{H} & 1.15455700 & -0.40311400 & 1.70824400 \\ \mathrm{C} & 2.19456000 & -1.57022500 & 0.21914300 \\ \mathrm{H} & 2.78934900 & -2.13325800 & 0.93700900 \\ \mathrm{H} & 1.96481400 & -2.22965400 & -0.61995300 \\ \mathrm{H} & 2.80470000 & -0.74298300 & -0.14804800 \\ \mathrm{O} & 1.89134600 & -0.75170500 & -2.65987300 \\ \mathrm{H} & 2.82529600 & -1.02217600 & -2.68264100\end{array}$

\section{PSIA••OH (TS5)}

$\begin{array}{lrrr}\mathrm{S} & 0.08091300 & 0.04404400 & -0.00054200 \\ \mathrm{O} & 0.07718000 & 0.12407900 & 1.47082400 \\ \mathrm{C} & 1.81837800 & -0.03649300 & -0.47691300 \\ \mathrm{H} & 1.86483000 & -0.01943600 & -1.56537600 \\ \mathrm{H} & 2.26272900 & 0.87429800 & -0.07243200 \\ \mathrm{O} & -0.07094900 & 1.57502900 & -0.61448800 \\ \mathrm{H} & -1.00833200 & 1.80062000 & -0.63469100\end{array}$




$\begin{array}{rrr}2.45393100 & -1.29062300 & 0.10068100 \\ 1.93856700 & -2.17692500 & -0.27516900 \\ 2.32961500 & -1.28353200 & 1.18618000 \\ 3.92416600 & -1.37049000 & -0.24800900 \\ 4.40497200 & -2.28191200 & 0.10073100 \\ 4.00497700 & -1.41082800 & -1.41032200 \\ 4.48593500 & -0.49492300 & 0.07433900 \\ 3.94400900 & -1.21960200 & -2.81959000 \\ 4.65049000 & -0.55709300 & -2.88066500\end{array}$

\section{PSIA ••OH (PC5)}

$\begin{array}{lccc}\mathrm{S} & -1.33804300 & 0.22744300 & 0.04428800 \\ \mathrm{O} & -1.72718700 & -0.06038100 & 1.43668600 \\ \mathrm{C} & 0.46417700 & 0.30020800 & 0.04872300 \\ \mathrm{H} & 0.77925500 & 0.56788100 & -0.95872700 \\ \mathrm{H} & 0.71268300 & 1.09699700 & 0.75104800 \\ \mathrm{O} & -1.45400500 & 1.86053700 & -0.20985900 \\ \mathrm{H} & -2.36773200 & 2.06500600 & -0.44018500 \\ \mathrm{C} & 1.02737700 & -1.04482600 & 0.48553400 \\ \mathrm{H} & 0.70587400 & -1.82603200 & -0.20869000 \\ \mathrm{H} & 0.59257300 & -1.30099200 & 1.46024500 \\ \mathrm{C} & 2.51152500 & -1.02491100 & 0.57454500 \\ \mathrm{H} & 3.06055200 & -1.95281700 & 0.64084000 \\ \mathrm{H} & 2.58577600 & -0.99009500 & -1.79783500 \\ \mathrm{H} & 3.02579500 & -0.11734500 & 0.85799700 \\ \mathrm{O} & 1.96763600 & -0.91452100 & -2.53336000 \\ \mathrm{H} & 2.48789100 & -1.04768200 & -3.32846900\end{array}$

\section{PSIA ••OH (TS6)}

$\begin{array}{lrrrr}\mathrm{S} & & 0.64761800 & 0.31390800 & 0.58482100 \\ \mathrm{O} & & 1.59495300 & 0.72505200 & 1.64568900 \\ \mathrm{C} & & 1.67641800 & -0.31874200 & -0.75647800 \\ \mathrm{H} & & 1.00418100 & -0.55613500 & -1.57822100\end{array}$




$\begin{array}{rrr}2.30147100 & 0.53133300 & -1.03528600 \\ 0.22541100 & 1.64931900 & -0.28354000 \\ -0.48003600 & 2.11178900 & 0.18501100 \\ 2.50693000 & -1.51377300 & -0.26923800 \\ 2.21915000 & -2.40807600 & -0.82432700 \\ 2.29100600 & -1.72106300 & 0.78099700 \\ 3.99253800 & -1.26978900 & -0.41973900 \\ 4.60823800 & -2.05267500 & 0.01618400 \\ 4.29324600 & -1.05918800 & -1.44478000 \\ 4.28664300 & -0.27129800 & 0.17415300 \\ 4.35283000 & 0.91327900 & 0.78744100 \\ 3.46635000 & 0.91112200 & 1.20390700\end{array}$

\section{PSIA ••OH (PC6)}

\begin{tabular}{lrrr}
\hline $\mathrm{S}$ & -1.28473900 & 0.13690400 & 0.17878300 \\
$\mathrm{O}$ & -0.56227700 & 0.44305900 & 1.43758700 \\
$\mathrm{C}$ & -0.01759500 & -0.40303800 & -0.98596500 \\
$\mathrm{H}$ & -0.49940900 & -0.49895800 & -1.95785800 \\
$\mathrm{H}$ & 0.71486000 & 0.40456600 & -1.00565200 \\
$\mathrm{O}$ & -1.52700900 & 1.55465500 & -0.63015700 \\
$\mathrm{H}$ & -2.32095000 & 1.97529100 & -0.27891600 \\
$\mathrm{C}$ & 0.61358600 & -1.71695400 & -0.50921500 \\
$\mathrm{H}$ & -0.12960800 & -2.51507300 & -0.54798800 \\
$\mathrm{H}$ & 0.91931100 & -1.58601400 & 0.53041800 \\
$\mathrm{C}$ & 1.79956300 & -2.05085300 & -1.34053900 \\
$\mathrm{H}$ & 2.71299900 & -1.49523800 & -1.19392700 \\
$\mathrm{H}$ & 1.72995300 & -2.73844100 & -2.16868100 \\
$\mathrm{H}$ & 2.77014700 & 1.11804900 & 1.49603600 \\
$\mathrm{O}$ & 2.21366200 & 0.57615800 & 0.93452900 \\
$\mathrm{H}$ & 1.31373100 & 0.65367700 & 1.29132900
\end{tabular}




\section{PSIA $\bullet$ OH (RC4)}

$\begin{array}{lrrr}\mathrm{S} & -0.86795700 & -0.51023500 & -0.48847200 \\ \mathrm{O} & -1.24478900 & -0.42530900 & 0.95327900 \\ \mathrm{C} & 0.89432900 & -0.10137600 & -0.50065000 \\ \mathrm{H} & 1.18225100 & -0.00301100 & -1.54809100 \\ \mathrm{H} & 0.98013200 & 0.87317300 & -0.01047000 \\ \mathrm{O} & -1.42094200 & 0.82114500 & -1.23483500 \\ \mathrm{H} & -1.24620900 & 1.59798100 & -0.66647000 \\ \mathrm{C} & 1.69580300 & -1.17155000 & 0.22356000 \\ \mathrm{H} & 1.52959800 & -2.13805700 & -0.25764300 \\ \mathrm{H} & 1.32439100 & -1.25596600 & 1.24501100 \\ \mathrm{C} & 3.18397100 & -0.84186200 & 0.22614600 \\ \mathrm{H} & 3.75096700 & -1.60893600 & 0.75073900 \\ \mathrm{H} & 3.57165300 & -0.77145400 & -0.79043400 \\ \mathrm{H} & 3.36802200 & 0.11144100 & 0.72190100 \\ \mathrm{O} & -0.73623800 & 2.22975900 & 1.16206200 \\ \mathrm{H} & -1.07064500 & 1.34950200 & 1.46254600\end{array}$

\section{PSIA $\bullet \circ H$ (TS7)}

$\begin{array}{lrrr}\mathrm{S} & -0.34967000 & -0.39676800 & -0.68080100 \\ \mathrm{O} & -0.69518000 & -0.37390800 & 0.77142300 \\ \mathrm{C} & 1.42860400 & -0.06543600 & -0.70865500 \\ \mathrm{H} & 1.69703700 & 0.06150800 & -1.75787600 \\ \mathrm{H} & 1.51103400 & 0.89183900 & -0.18558500 \\ \mathrm{O} & -0.89708200 & 0.92846500 & -1.30649300 \\ \mathrm{H} & -0.84017900 & 1.67206900 & -0.49743000 \\ \mathrm{C} & 2.21006200 & -1.17634600 & -0.03005000 \\ \mathrm{H} & 2.02661400 & -2.12223700 & -0.54400200 \\ \mathrm{H} & 1.84512300 & -1.28904800 & 0.99079700 \\ \mathrm{C} & 3.70384400 & -0.86926900 & -0.02667000 \\ \mathrm{H} & 4.26148400 & -1.66322000 & 0.46703500 \\ \mathrm{H} & 4.08585400 & -0.76961100 & -1.04288600\end{array}$




$\begin{array}{lrrr}\mathrm{H} & 3.90551000 & 0.06316600 & 0.50053600 \\ \mathrm{O} & -0.53033400 & 2.21595300 & 0.67875500 \\ \mathrm{H} & -0.95043000 & 1.67878000 & 1.37013100\end{array}$

\section{PSIA $\cdot 0$ OH (PC7)}

$\begin{array}{lrrr}\mathrm{S} & -0.88056400 & -0.46286400 & -0.51946100 \\ \mathrm{O} & -1.28927300 & -0.68403000 & 0.87114800 \\ \mathrm{C} & 0.91563300 & -0.11620200 & -0.51947000 \\ \mathrm{H} & 1.17997600 & -0.03728000 & -1.57252800 \\ \mathrm{H} & 0.95471200 & 0.86650600 & -0.04413200 \\ \mathrm{O} & -1.52981100 & 0.59534800 & -1.29779900 \\ \mathrm{H} & -1.26864200 & 2.34159900 & 0.29611400 \\ \mathrm{C} & 1.69123500 & -1.18230100 & 0.22782900 \\ \mathrm{H} & 1.53552500 & -2.15370200 & -0.24425700 \\ \mathrm{H} & 1.30698000 & -1.25285000 & 1.24460300 \\ \mathrm{C} & 3.17779800 & -0.83971500 & 0.24409400 \\ \mathrm{H} & 3.73982300 & -1.59819500 & 0.78589100 \\ \mathrm{H} & 3.57889300 & -0.78073700 & -0.76781400 \\ \mathrm{H} & 3.34887800 & 0.12015500 & 0.73099400 \\ \mathrm{O} & -0.71117700 & 2.24966800 & 1.07550300 \\ \mathrm{H} & -1.13368100 & 1.55177600 & 1.58654100\end{array}$

\section{$\underline{\mathrm{CH}_{3}} \underline{-\mathrm{CH}_{2}} \underline{\underline{\mathrm{CH}}} \underline{2} \underline{2}-\mathrm{S}(\mathrm{O}) \mathrm{O} \bullet$ (P4)}

$\begin{array}{lrrr}\mathrm{S} & -0.88657500 & -0.46615100 & -0.51455000 \\ \mathrm{O} & -1.28366300 & -0.68156500 & 0.87681700 \\ \mathrm{C} & 0.90952200 & -0.11188600 & -0.51477200 \\ \mathrm{H} & 1.17523800 & -0.02745400 & -1.56689800 \\ \mathrm{H} & 0.97235800 & 0.87088500 & -0.04459900 \\ \mathrm{O} & -1.52831300 & 0.57507100 & -1.31559600 \\ \mathrm{C} & 1.68971200 & -1.17764800 & 0.22884600 \\ \mathrm{H} & 1.52982100 & -2.14608800 & -0.24793600 \\ \mathrm{H} & 1.30497800 & -1.25387300 & 1.24502100\end{array}$




$\begin{array}{llrc}\mathrm{C} & 3.17721200 & -0.84095000 & 0.24406300 \\ \mathrm{H} & 3.73810200 & -1.60436100 & 0.78007200 \\ \mathrm{H} & 3.57669700 & -0.77693300 & -0.76819300 \\ \mathrm{H} & 3.35471800 & 0.11508600 & 0.73682400\end{array}$

\section{PSIA••OH (RC5)}

$\begin{array}{lccc}\mathrm{S} & -0.56659200 & -0.40048300 & -0.50737400 \\ \mathrm{O} & -0.79721700 & -0.65337800 & 0.94297200 \\ \mathrm{C} & 1.12983300 & 0.18813500 & -0.62239600 \\ \mathrm{H} & 1.26899600 & 0.54038700 & -1.64499600 \\ \mathrm{H} & 1.18074200 & 1.03323900 & 0.06532400 \\ \mathrm{O} & -1.20242000 & 1.06983400 & -0.85720600 \\ \mathrm{H} & -2.15165100 & 1.02728100 & -0.64114800 \\ \mathrm{O} & -3.38111500 & 0.39674900 & 0.88134700 \\ \mathrm{H} & -2.58450500 & -0.06613500 & 1.23156100 \\ \mathrm{C} & 2.09713300 & -0.92449000 & -0.25086100 \\ \mathrm{H} & 1.85861800 & -1.27517300 & 0.75361400 \\ \mathrm{H} & 1.95999900 & -1.77114500 & -0.92703800 \\ \mathrm{C} & 3.54085600 & -0.43979700 & -0.31234400 \\ \mathrm{H} & 4.23048900 & -1.23687300 & -0.04009900 \\ \mathrm{H} & 3.79756900 & -0.09924100 & -1.31577400 \\ \mathrm{H} & 3.69908000 & 0.39190600 & 0.37437600\end{array}$

\section{PSIA • OH (TS8)}

$\begin{array}{lccc}\mathrm{S} & 0.37866400 & -0.85277400 & 0.40202200 \\ \mathrm{O} & 0.44220900 & -0.88860600 & 1.87377600 \\ \mathrm{C} & 2.07172400 & -0.57014200 & -0.16243900 \\ \mathrm{H} & 2.02500600 & -0.46098800 & -1.24571900 \\ \mathrm{H} & 2.35597100 & 0.38239200 & 0.28794500 \\ \mathrm{O} & -0.13904900 & 0.61379500 & -0.09503900 \\ \mathrm{H} & -1.10561500 & 0.54534100 & -0.12997300 \\ \mathrm{O} & -2.05624500 & -1.38553700 & 0.19937200\end{array}$




$\begin{array}{rrr}-1.96647600 & -1.71850000 & 1.10959200 \\ 2.97244400 & -1.71118800 & 0.27764100 \\ 2.91353300 & -1.80435800 & 1.36204100 \\ 2.60797500 & -2.65003200 & -0.14497900 \\ 4.41350100 & -1.47073700 & -0.15730300 \\ 5.05748300 & -2.28738500 & 0.16446100 \\ 4.48806300 & -1.38942600 & -1.24201600 \\ 4.79918400 & -0.54729200 & 0.27511000\end{array}$

\section{PSIA $\bullet(\mathrm{OH})_{2}(\mathrm{PC} 8)$}

$\begin{array}{lrrr}\mathrm{S} & -0.72920500 & 0.01001500 & 0.05214000 \\ \mathrm{O} & -0.56207200 & 0.03837600 & 1.50222600 \\ \mathrm{C} & 1.07211800 & 0.30932000 & -0.55249200 \\ \mathrm{H} & 0.99993600 & 0.39500300 & -1.63499100 \\ \mathrm{H} & 1.32365700 & 1.26980100 & -0.10408000 \\ \mathrm{O} & -1.17402700 & 1.43757400 & -0.55805000 \\ \mathrm{H} & -2.13672000 & 1.49435200 & -0.46618900 \\ \mathrm{O} & -2.46474900 & -0.48718600 & -0.08723800 \\ \mathrm{H} & -2.57350500 & -1.18288900 & 0.57300600 \\ \mathrm{C} & 1.94023400 & -0.83312500 & -0.08386900 \\ \mathrm{H} & 1.88225100 & -0.90088700 & 1.00244900 \\ \mathrm{H} & 1.55638000 & -1.77355600 & -0.48597100 \\ \mathrm{C} & 3.38939400 & -0.63895600 & -0.52350500 \\ \mathrm{H} & 4.01176500 & -1.46459100 & -0.18149600 \\ \mathrm{H} & 3.46571100 & -0.58521800 & -1.60970300 \\ \mathrm{H} & 3.79864200 & 0.28406500 & -0.11274400\end{array}$

\section{PSIA ••OH (TS9)}

S $\quad-0.72289500 \quad-0.05836500 \quad-0.00678000$

$\begin{array}{llll}\mathrm{O} & -0.46231700 & 0.03800200 & 1.42647100\end{array}$

$\begin{array}{llll}\mathrm{C} & 1.28704600 & 0.35220800 & -0.67423500\end{array}$

$\begin{array}{lllll}\mathrm{H} & 1.16167100 & 0.45713700 & -1.74777400\end{array}$ 


$\begin{array}{rrr}1.43040900 & 1.29812700 & -0.16036000 \\ -1.13005700 & 1.37821700 & -0.63821700 \\ -2.06135500 & 1.53800900 & -0.42555500 \\ -2.38914700 & -0.51370300 & -0.02141200 \\ -2.50953900 & -1.08272000 & 0.74938500 \\ 2.11930600 & -0.79898700 & -0.20228900 \\ 2.00390500 & -0.90654100 & 0.87688800 \\ 1.76405900 & -1.72418200 & -0.66118100 \\ 3.59704500 & -0.59393700 & -0.54807200 \\ 4.19435700 & -1.43609900 & -0.20024500 \\ 3.73675500 & -0.49959200 & -1.62491100 \\ 3.98177400 & 0.31120100 & -0.07872800\end{array}$

\section{$\underline{\mathbf{H}}_{2} \underline{\mathrm{SO}}_{3}$}

$\begin{array}{lrrr}{ }_{\mathrm{S}} \underline{\mathrm{SO}} & -0.76657300 & -0.05699800 & -0.01024800 \\ \mathrm{O} & -0.68767900 & 0.33069900 & 1.39651700 \\ \mathrm{O} & -1.38490200 & 1.23449200 & -0.77063200 \\ \mathrm{H} & -1.82113000 & 1.79838100 & -0.11222100 \\ \mathrm{O} & -2.02728200 & -1.07229300 & -0.09990300 \\ \mathrm{H} & -2.58774300 & -0.93484100 & 0.68037900\end{array}$

\section{$\mathrm{CH}_{3} \underline{\mathrm{CH}}_{2} \underline{\mathrm{C} \cdot \mathrm{H}_{2}}$}

\begin{tabular}{|c|c|c|c|}
\hline $\mathrm{C}$ & 1.35568700 & 0.40191000 & -0.71194400 \\
\hline $\mathrm{H}$ & 1.01484400 & 0.44597500 & -1.73565300 \\
\hline $\mathrm{H}$ & 1.27347100 & 1.29917200 & -0.11675900 \\
\hline $\mathrm{C}$ & 2.14369200 & -0.76051400 & -0.22525600 \\
\hline $\mathrm{H}$ & 2.02074400 & -0.86953700 & 0.85408400 \\
\hline $\mathrm{H}$ & 1.77259900 & -1.68053800 & -0.68113900 \\
\hline $\mathrm{C}$ & 3.64229100 & -0.62290900 & -0.54037800 \\
\hline $\mathrm{H}$ & 4.19984500 & -1.48559100 & -0.17482300 \\
\hline $\mathrm{H}$ & 3.80179400 & -0.54159900 & -1.61544500 \\
\hline $\mathrm{H}$ & 4.05135900 & 0.27296700 & -0.07359400 \\
\hline
\end{tabular}




\section{PSIA $\bullet O H$ (TS10)}

$\begin{array}{lrrr}\mathrm{S} & -0.44996800 & 0.26139700 & 0.71896300 \\ \mathrm{O} & -0.05749700 & 0.54425700 & 2.10307500 \\ \mathrm{C} & 1.03894700 & 0.33540200 & -0.29762800 \\ \mathrm{H} & 0.67427900 & 0.28061100 & -1.32096000 \\ \mathrm{H} & 1.45972200 & 1.32027800 & -0.08962400 \\ \mathrm{O} & -1.36815500 & 1.39165300 & 0.15415200 \\ \mathrm{H} & -1.84023600 & 0.69689800 & -0.53977300 \\ \mathrm{O} & -1.65655900 & -0.63274900 & -0.95431800 \\ \mathrm{H} & -2.28441900 & -1.28381800 & -0.62634100 \\ \mathrm{C} & 1.98209500 & -0.80072000 & 0.05634300 \\ \mathrm{H} & 2.22659500 & -0.74707600 & 1.11739900 \\ \mathrm{H} & 1.47323100 & -1.75094100 & -0.11512700 \\ \mathrm{C} & 3.25161300 & -0.72789600 & -0.78463600 \\ \mathrm{H} & 3.92752500 & -1.54317700 & -0.53345400 \\ \mathrm{H} & 3.01987700 & -0.79624200 & -1.84745900 \\ \mathrm{H} & 3.77816300 & 0.21120700 & -0.61383900\end{array}$

\section{PSIA ••OH (TS11)}

$\begin{array}{lrrr}\mathrm{S} & -0.37124300 & -0.13497200 & 0.03216600 \\ \mathrm{O} & -0.37972900 & -1.23723000 & 0.96646900 \\ \mathrm{C} & 1.71740600 & 0.00776700 & -0.64408300 \\ \mathrm{H} & 1.57392500 & -0.19922300 & -1.70222100 \\ \mathrm{H} & 2.28756700 & 0.96133700 & -0.60402300 \\ \mathrm{O} & -0.13703300 & 1.22121000 & 0.72239500 \\ \mathrm{H} & 0.95819300 & 0.91277000 & 0.02253400 \\ \mathrm{C} & 2.44409800 & -1.06687500 & 0.13541900 \\ \mathrm{H} & 1.89903300 & -2.00665700 & 0.03651100 \\ \mathrm{H} & 2.41499000 & -0.81544200 & 1.19744800 \\ \mathrm{C} & 3.88822800 & -1.25109600 & -0.32219200 \\ \mathrm{H} & 4.37658200 & -2.04451800 & 0.24185700 \\ \mathrm{H} & 3.92982700 & -1.51379100 & -1.37971100\end{array}$




\section{PSIA・・OH (PC9)}

$\begin{array}{lrrr}\mathrm{S} & -1.64242900 & -0.27191200 & -0.20846800 \\ \mathrm{O} & -1.11132100 & -1.60000100 & -0.34787300 \\ \mathrm{C} & 1.36515600 & 0.89553600 & -0.87875800 \\ \mathrm{H} & 0.72430000 & 0.36752500 & -1.59085300 \\ \mathrm{H} & 2.17972000 & 1.32942300 & -1.46043700 \\ \mathrm{O} & -1.54159500 & 0.35529700 & 1.08021700 \\ \mathrm{H} & 0.79699900 & 1.71601400 & -0.43842400 \\ \mathrm{C} & 1.90445300 & -0.05898800 & 0.17973700 \\ \mathrm{H} & 1.09414300 & -0.40024100 & 0.82704900 \\ \mathrm{H} & 2.60037800 & 0.47930000 & 0.82549600 \\ \mathrm{C} & 2.59670300 & -1.26410900 & -0.44270600 \\ \mathrm{H} & 3.00262000 & -1.93028200 & 0.31742100 \\ \mathrm{H} & 1.89307700 & -1.83704100 & -1.04866400 \\ \mathrm{H} & 3.41758900 & -0.95033900 & -1.08963400 \\ & & & \\ \mathrm{SO}_{2} & & & \\ \mathrm{~S} & -1.64131500 & -0.27322700 & -0.20656200 \\ \mathrm{O} & -1.11163100 & -1.60049200 & -0.34968500 \\ \mathrm{O} & -1.54239900 & 0.35710300 & 1.08012300\end{array}$

\section{$\underline{\mathbf{C}}_{3} \underline{\underline{H}}_{6}$}

$\begin{array}{lrrr}\mathrm{C} & 1.35815000 & 0.89180400 & -0.88201700 \\ \mathrm{H} & 0.64924900 & 0.38129700 & -1.53591000 \\ \mathrm{H} & 2.16743500 & 1.27231200 & -1.50733800 \\ \mathrm{H} & 0.84867400 & 1.74570700 & -0.43631000 \\ \mathrm{C} & 1.89770900 & -0.06135000 & 0.17695700 \\ \mathrm{H} & 1.07726500 & -0.41025900 & 0.80712800 \\ \mathrm{H} & 2.58444000 & 0.47426900 & 0.83534400\end{array}$




$\begin{array}{lrrr}\mathrm{C} & 2.61125300 & -1.25758500 & -0.44032600 \\ \mathrm{H} & 2.99556300 & -1.93649800 & 0.32041900 \\ \mathrm{H} & 1.93359300 & -1.82195900 & -1.08296900 \\ \mathrm{H} & 3.45180700 & -0.93093900 & -1.05475200\end{array}$

Table S6: T1 diagnostic values for the various conformers, and all other stationary points involved in the PSIA $+{ }^{\circ} \mathrm{OH}$ reaction system calculated at the CCSD(T)/aug-cc-pVTZ level.

\begin{tabular}{|c|c|c|c|}
\hline species & T1 value & species & T1 value \\
\hline PSIA-I & 0.015 & TS5 & 0.019 \\
\hline PSIA-II & 0.015 & PC5 & 0.015 \\
\hline PSIA-III & 0.015 & RC3 & 0.015 \\
\hline PSIA-IV & 0.015 & TS6 & 0.019 \\
\hline PSIA-V & 0.015 & PC6 & 0.015 \\
\hline $\mathrm{RC} 1$ & 0.015 & $\mathrm{RC} 4$ & 0.016 \\
\hline TS1 & 0.019 & TS7 & 0.016 \\
\hline $\mathrm{PC} 1$ & 0.017 & PC7 & 0.017 \\
\hline P1 & 0.018 & P4 & 0.018 \\
\hline $\mathrm{RC} 2$ & 0.015 & TS8 & 0.019 \\
\hline TS2 & 0.015 & $\mathrm{RC} 5$ & 0.015 \\
\hline PC2 & 0.017 & PC8 & 0.018 \\
\hline TS3 & 0.019 & TS9 & 0.018 \\
\hline PC3 & 0.016 & TS10 & 0.027 \\
\hline $\mathrm{P} 2$ & 0.018 & $\mathrm{C}_{3} \mathrm{H}_{7}$ & 0.011 \\
\hline TS4 & 0.019 & $\mathrm{H}_{2} \mathrm{SO}_{3}$ & 0.017 \\
\hline PC4 & 0.015 & $\mathrm{OH}$ & 0.01 \\
\hline P3 & 0.016 & $\mathrm{H}_{2} \mathrm{O}$ & 0.01 \\
\hline $\mathrm{SO}_{2}$ & 0.021 & & \\
\hline
\end{tabular}


Table S7: Calculated Eckart tunneling contributions for all the possible H-atom abstraction, and ${ }^{\circ}$ OH addition pathways involved in the PSIA + $\cdot \mathrm{OH}$ reaction in the temperatures between 200 and $320 \mathrm{~K}$ and at 1 atm pressure.

\begin{tabular}{|c|c|c|c|c|c|c|c|c|}
\hline $\mathrm{T}(\mathrm{K})$ & $\mathrm{k}_{\mathrm{TS} 1}$ & $\mathrm{k}_{\mathrm{TS} 2}$ & $\mathrm{k}_{\mathrm{TS} 3}$ & $\mathrm{k}_{\mathrm{TS} 4}$ & $\mathrm{k}_{\mathrm{TS} 5}$ & $\mathrm{k}_{\mathrm{TS} 6}$ & $\mathrm{k}_{\mathrm{TS} 7}$ & $\mathrm{k}_{\mathrm{TS} 8}$ \\
\hline 200 & 2.4 & 3.2 & 1.0 & 5.7 & 2.0 & 1.0 & 9.2 & 4.0 \\
\hline 220 & 2.4 & 3.0 & 1.0 & 4.1 & 1.9 & 1.0 & 9.0 & 3.0 \\
\hline 240 & 2.4 & 2.8 & 1.0 & 3.2 & 1.8 & 1.0 & 8.6 & 3.0 \\
\hline 250 & 2.4 & 2.6 & 1.0 & 2.9 & 1.8 & 1.0 & 8.4 & 3.0 \\
\hline 260 & 2.4 & 2.5 & 1.0 & 2.7 & 1.7 & 1.0 & 8.2 & 3.0 \\
\hline 280 & 2.3 & 2.4 & 1.0 & 2.3 & 1.6 & 1.0 & 7.8 & 2.0 \\
\hline 298 & 2.1 & 2.3 & 1.0 & 2.1 & 1.6 & 1.0 & 7.5 & 2.0 \\
\hline 320 & 2.0 & 2.2 & 1.0 & 1.9 & 1.5 & 1.0 & 7.0 & 2.0 \\
\hline
\end{tabular}

Table S8: Calculated bimolecular rate coefficients (in $\mathrm{cm}^{3}$ molecule ${ }^{-1} \mathrm{~s}^{-1}$ ) for all the possible $\mathrm{H}$-atom abstraction, and ${ }^{\bullet} \mathrm{OH}$ addition pathways involved in the PSIA + ${ }^{\circ} \mathrm{OH}$ reaction in the temperatures between 200 and $320 \mathrm{~K}$ and at 0.1 atm pressure.

\begin{tabular}{|c|c|c|c|c|c|c|c|c|c|}
\hline $\mathrm{T}(\mathrm{K})$ & $k_{\mathrm{TS} 1}$ & $k_{\mathrm{TS} 2}$ & $k_{\mathrm{TS} 3}$ & $k_{\mathrm{TS} 4}$ & $k_{\mathrm{TS} 5}$ & $k_{\mathrm{TS} 6}$ & $k_{\mathrm{TS} 7}$ & $k_{\mathrm{TS} 8}$ & $k_{\text {total }}{ }^{\mathrm{a}}$ \\
\hline 200 & $5.41 \times 10^{-13}$ & $4.41 \times 10^{-12}$ & $7.17 \times 10^{-12}$ & $6.63 \times 10^{-15}$ & $2.79 \times 10^{-14}$ & $8.34 \times 10^{-13}$ & $9.35 \times 10^{-11}$ & $6.31 \times 10^{-12}$ & $1.20 \times 10^{-10}$ \\
\hline 220 & $4.65 \times 10^{-13}$ & $3.72 \times 10^{-12}$ & $6.52 \times 10^{-12}$ & $8.81 \times 10^{-15}$ & $3.44 \times 10^{-14}$ & $5.60 \times 10^{-13}$ & $8.93 \times 10^{-11}$ & $3.48 \times 10^{-12}$ & $1.11 \times 10^{-10}$ \\
\hline 240 & $4.15 \times 10^{-13}$ & $3.14 \times 10^{-12}$ & $5.92 \times 10^{-12}$ & $1.18 \times 10^{-14}$ & $4.23 \times 10^{-14}$ & $3.96 \times 10^{-13}$ & $8.47 \times 10^{-11}$ & $1.91 \times 10^{-12}$ & $1.02 \times 10^{-10}$ \\
\hline 250 & $3.97 \times 10^{-13}$ & $2.90 \times 10^{-12}$ & $5.63 \times 10^{-12}$ & $1.36 \times 10^{-14}$ & $4.67 \times 10^{-14}$ & $3.40 \times 10^{-13}$ & $8.23 \times 10^{-11}$ & $1.42 \times 10^{-12}$ & $9.86 \times 10^{-11}$ \\
\hline 260 & $3.83 \times 10^{-13}$ & $2.67 \times 10^{-12}$ & $5.36 \times 10^{-12}$ & $1.56 \times 10^{-14}$ & $5.15 \times 10^{-14}$ & $2.96 \times 10^{-13}$ & $7.97 \times 10^{-11}$ & $1.06 \times 10^{-12}$ & $9.49 \times 10^{-11}$ \\
\hline 280 & $3.62 \times 10^{-13}$ & $2.29 \times 10^{-12}$ & $4.85 \times 10^{-12}$ & $2.05 \times 10^{-14}$ & $6.22 \times 10^{-14}$ & $2.33 \times 10^{-13}$ & $7.45 \times 10^{-11}$ & $6.04 \times 10^{-13}$ & $8.78 \times 10^{-11}$ \\
\hline 298 & $3.51 \times 10^{-13}$ & $2.00 \times 10^{-12}$ & $4.44 \times 10^{-12}$ & $2.59 \times 10^{-14}$ & $7.31 \times 10^{-14}$ & $1.95 \times 10^{-13}$ & $6.98 \times 10^{-11}$ & $3.73 \times 10^{-13}$ & $8.17 \times 10^{-11}$ \\
\hline 320 & $3.45 \times 10^{-13}$ & $1.72 \times 10^{-12}$ & $3.99 \times 10^{-12}$ & $3.39 \times 10^{-14}$ & $8.81 \times 10^{-14}$ & $1.64 \times 10^{-13}$ & $6.39 \times 10^{-11}$ & $2.16 \times 10^{-13}$ & $7.45 \times 10^{-11}$ \\
\hline
\end{tabular}

${ }^{a}$ The overall rate coefficient $\left(k_{\text {total }}\right)$ for the PSIA $+{ }^{\circ} \mathrm{OH}$ reaction was calculated by the summing of all the individual reaction pathways at the corresponding temperatures. The contribution of the rate coefficient via $\mathrm{k}_{\mathrm{TS} 3}$ was multiplied by a factor of 2 . 
Table S9: Calculated bimolecular rate coefficients (in $\mathrm{cm}^{3}$ molecule ${ }^{-1} \mathrm{~s}^{-1}$ ) for all the possible $\mathrm{H}$-atom abstraction, and ${ }^{\cdot} \mathrm{OH}$ addition pathways involved in the PSIA + ${ }^{\circ} \mathrm{OH}$ reaction in the temperatures between 200 and $320 \mathrm{~K}$ and at 10 atm pressure.

\begin{tabular}{|c|c|c|c|c|c|c|c|c|c|}
\hline $\mathrm{T}(\mathrm{K})$ & $k_{\mathrm{TS} 1}$ & $k_{\mathrm{TS} 2}$ & $k_{\mathrm{TS} 3}$ & $k_{\mathrm{TS} 4}$ & $k_{\mathrm{TS} 5}$ & $k_{\mathrm{TS} 6}$ & $k_{\mathrm{TS} 7}$ & $k_{\mathrm{TS} 8}$ & $k_{\text {total }}{ }^{\mathrm{a}}$ \\
\hline 200 & $1.68 \times 10^{-12}$ & $6.50 \times 10^{-12}$ & $7.76 \times 10^{-12}$ & $4.32 \times 10^{-15}$ & $1.19 \times 10^{-13}$ & $5.72 \times 10^{-12}$ & $9.38 \times 10^{-11}$ & $6.67 \times 10^{-11}$ & $1.80 \times 10^{-10}$ \\
\hline 220 & $1.30 \times 10^{-12}$ & $5.39 \times 10^{-12}$ & $7.01 \times 10^{-12}$ & $6.91 \times 10^{-15}$ & $1.21 \times 10^{-13}$ & $4.37 \times 10^{-12}$ & $8.96 \times 10^{-11}$ & $4.33 \times 10^{-11}$ & $1.58 \times 10^{-10}$ \\
\hline 240 & $1.05 \times 10^{-12}$ & $4.42 \times 10^{-12}$ & $6.30 \times 10^{-12}$ & $1.03 \times 10^{-14}$ & $1.27 \times 10^{-13}$ & $3.22 \times 10^{-12}$ & $8.49 \times 10^{-11}$ & $3.17 \times 10^{-11}$ & $1.38 \times 10^{-10}$ \\
\hline 250 & $9.56 \times 10^{-13}$ & $4.00 \times 10^{-12}$ & $5.97 \times 10^{-12}$ & $1.23 \times 10^{-14}$ & $1.31 \times 10^{-13}$ & $2.75 \times 10^{-12}$ & $8.25 \times 10^{-11}$ & $2.68 \times 10^{-11}$ & $1.29 \times 10^{-10}$ \\
\hline 260 & $8.80 \times 10^{-13}$ & $3.63 \times 10^{-12}$ & $5.66 \times 10^{-12}$ & $1.45 \times 10^{-14}$ & $1.35 \times 10^{-13}$ & $2.34 \times 10^{-12}$ & $7.99 \times 10^{-11}$ & $2.24 \times 10^{-11}$ & $1.21 \times 10^{-10}$ \\
\hline 280 & $7.66 \times 10^{-13}$ & $3.01 \times 10^{-12}$ & $5.09 \times 10^{-12}$ & $1.97 \times 10^{-14}$ & $1.46 \times 10^{-13}$ & $1.70 \times 10^{-12}$ & $7.47 \times 10^{-11}$ & $1.55 \times 10^{-11}$ & $1.06 \times 10^{-10}$ \\
\hline 298 & $6.94 \times 10^{-13}$ & $2.57 \times 10^{-12}$ & $4.64 \times 10^{-12}$ & $2.53 \times 10^{-14}$ & $1.56 \times 10^{-13}$ & $1.29 \times 10^{-12}$ & $6.99 \times 10^{-11}$ & $1.11 \times 10^{-11}$ & $9.50 \times 10^{-11}$ \\
\hline 320 & $6.33 \times 10^{-13}$ & $2.15 \times 10^{-12}$ & $4.16 \times 10^{-12}$ & $3.35 \times 10^{-14}$ & $1.72 \times 10^{-13}$ & $9.37 \times 10^{-13}$ & $6.41 \times 10^{-11}$ & $7.38 \times 10^{-12}$ & $8.37 \times 10^{-11}$ \\
\hline
\end{tabular}

${ }^{a}$ The overall rate coefficient $\left(k_{\text {total }}\right)$ for the PSIA $+{ }^{\circ} \mathrm{OH}$ reaction was calculated by the summing of all the individual reaction pathways at the corresponding temperatures. The contribution of the rate coefficient via $\mathrm{k}_{\mathrm{TS} 3}$ was multiplied by a factor of 2 . 
Table S10: The calculated branching ratios for all the abstraction and addition paths involved in the PSIA + $\bullet \mathrm{OH}$ reaction in the temperatures between 200 and $320 \mathrm{~K}$ and at 760 Torr pressure.

\begin{tabular}{|c|c|c|c|c|c|c|c|c|}
\hline $\mathrm{T}(\mathrm{K})$ & $k_{\mathrm{TS} 1}$ & $k_{\mathrm{TS} 2}$ & $k_{\mathrm{TS} 3}$ & $k_{\mathrm{TS} 4}$ & $k_{\mathrm{TS} 5}$ & $k_{\mathrm{TS} 6}$ & $k_{\mathrm{TS} 7}$ & $k_{\mathrm{TS} 8}$ \\
\hline 200 & 0.54 & 3.6 & 5.5 & $4.54 \times 10^{-3}$ & $2.85 \times 10^{-2}$ & 1.7 & 70.7 & 17.9 \\
\hline 220 & 0.50 & 3.4 & 5.6 & $7.17 \times 10^{-3}$ & $3.73 \times 10^{-2}$ & 1.3 & 76.2 & 12.9 \\
\hline 240 & 0.49 & 3.2 & 5.7 & $1.10 \times 10^{-2}$ & $4.87 \times 10^{-2}$ & 1.0 & 80.7 & 8.9 \\
\hline 250 & 0.48 & 3.1 & 5.7 & $1.34 \times 10^{-2}$ & $5.56 \times 10^{-2}$ & 0.8 & 82.5 & 7.4 \\
\hline 260 & 0.48 & 2.9 & 5.7 & $1.63 \times 10^{-2}$ & $6.33 \times 10^{-2}$ & 0.7 & 84.0 & 6.1 \\
\hline 280 & 0.49 & 2.8 & 5.6 & $2.37 \times 10^{-2}$ & $8.18 \times 10^{-2}$ & 0.6 & 86.3 & 4.1 \\
\hline 298 & 0.51 & 2.6 & 5.6 & $3.25 \times 10^{-2}$ & $1.02 \times 10^{-1}$ & 0.5 & 87.7 & 2.9 \\
\hline 320 & 0.54 & 2.5 & 5.6 & $4.71 \times 10^{-2}$ & $1.34 \times 10^{-1}$ & 0.4 & 88.9 & 2.0 \\
\hline
\end{tabular}

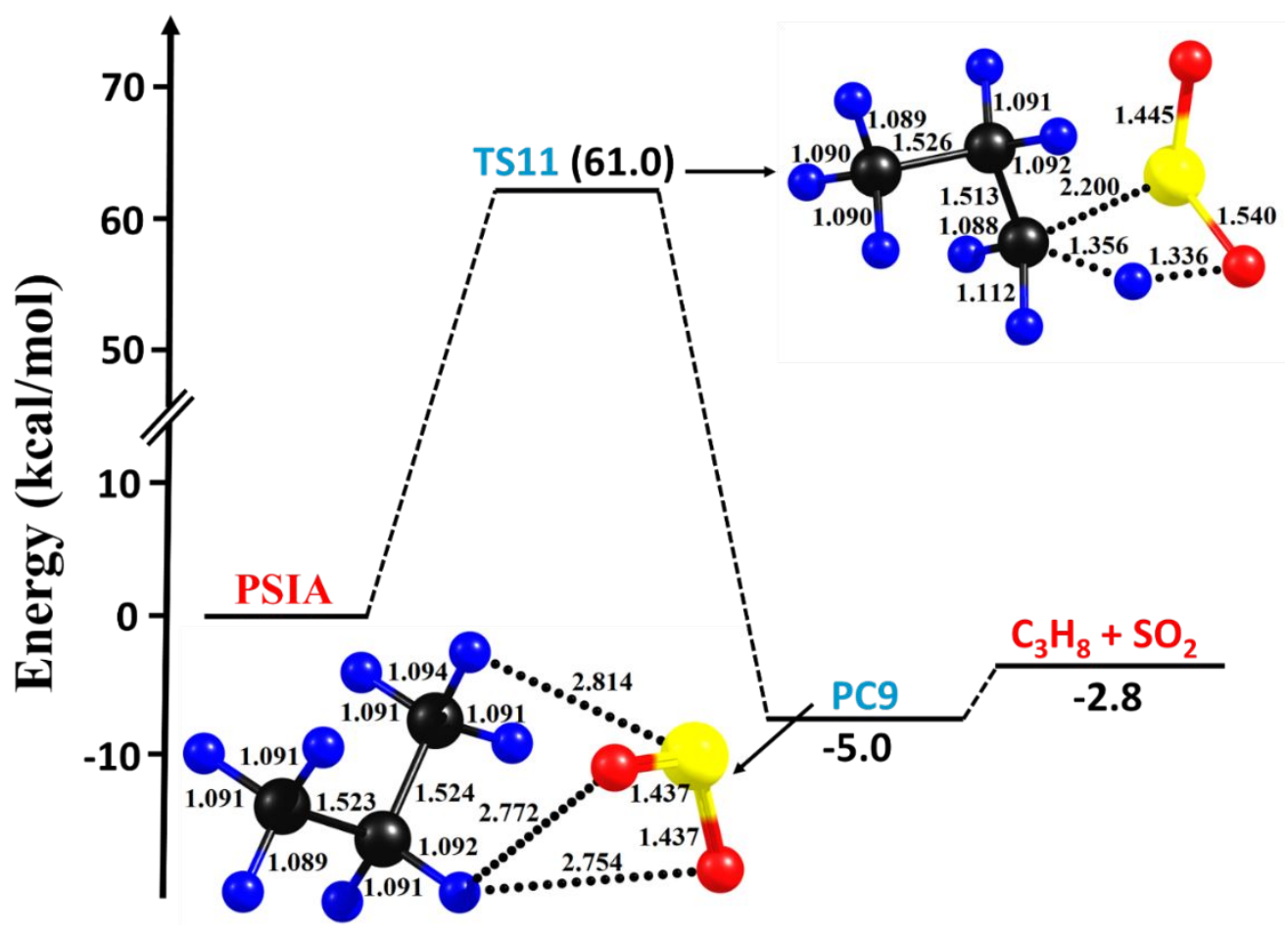

Figure S1. Potential energy profile for the direct elimination of sulfur dioxide $\left(\mathrm{SO}_{2}\right)$ from propanesulfinic acid to form propane $\left(\mathrm{C}_{3} \mathrm{H}_{8}\right)+\mathrm{SO}_{2}$ as products. The energies of the stationary points on the PES were calculated at the $\operatorname{CCSD}(\mathrm{T}) /$ aug-cc-pVTZ//M06-2X/aug-cc-pVTZ level. The relative energies include zero point corrections. The symbols TS11 and PC9 refer to the transition state and post-reactive complex respectively. 


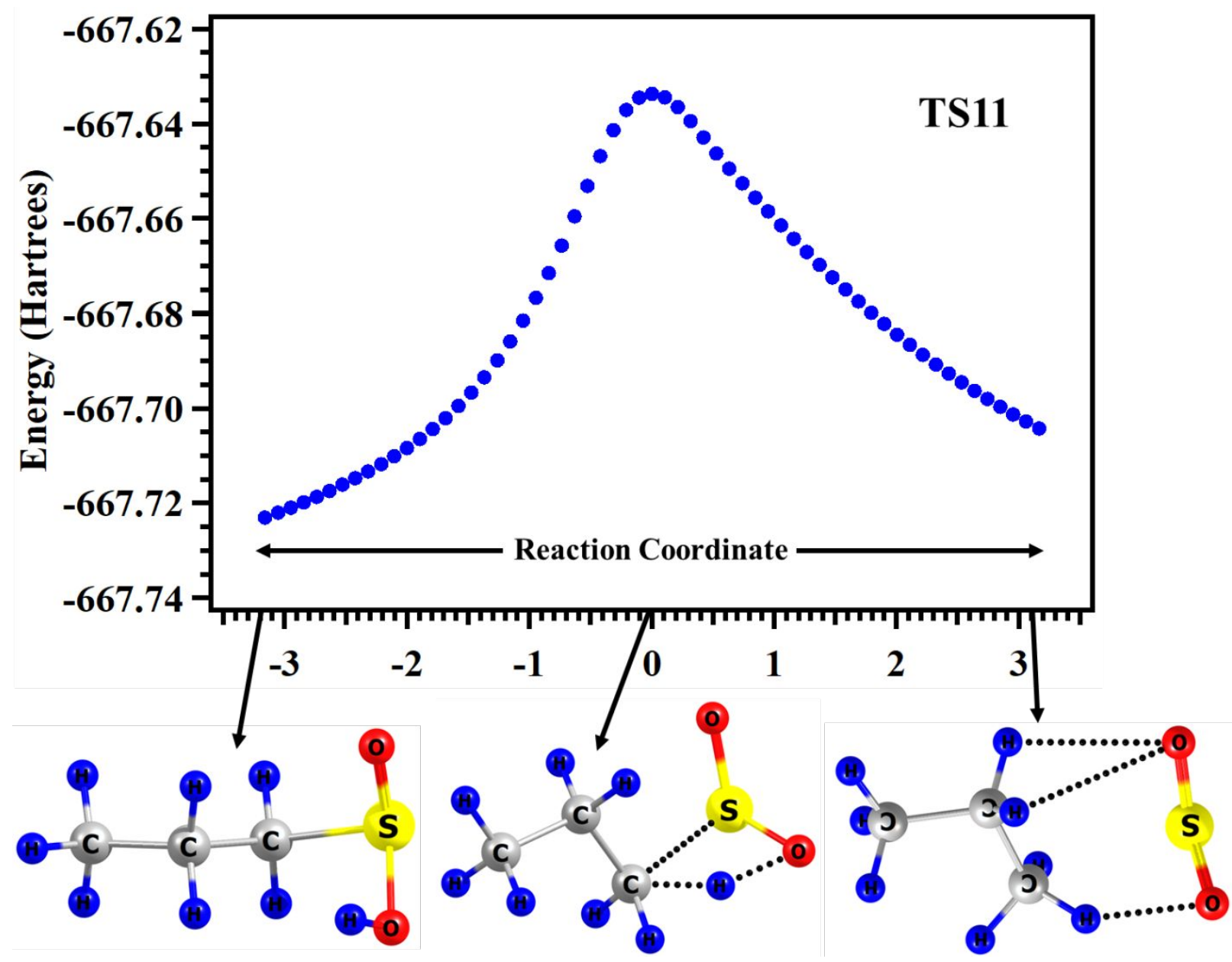

Figure S2. The M06-2X/aug-cc-pVTZ level calculated internal reaction coordinate (IRC) plot for the transition states (TS11) involving the direct elimination of $\mathrm{SO}_{2}$ to form propane from the unimolecular decomposition of PSIA. The reaction proceeds from reactant to post-reactive complex via a transition state, as indicated with the various structures (left to right) on the reaction coordinate. The yellow, black, blue, and red colors represent sulfur, carbon, hydrogen, and oxygen atoms, respectively. 